\title{
TITLE:
}

\section{Critical correlation of bi-directional horizontal ground motions}

\author{
$\operatorname{AUTHOR}(\mathrm{S})$ :
}

Fujita, Kohei; Takewaki, Izuru

\section{CITATION:}

Fujita, Kohei ...[et al]. Critical correlation of bi-directional horizontal ground motions. Engineering Structures 2010, 32(1): 261-272

\section{ISSUE DATE:}

2010-01

URL:

http://hdl.handle.net/2433/88958

\section{RIGHT:}

c 2009 Elsevier Ltd. All rights reserved.; This is not the published version. Please cite only the published version.; この論文は出版社版でありませ ん。引用の際には出版社版をご確認ご利用ください。 


\title{
Critical correlation of bi-directional horizontal ground motions
}

\author{
K. Fujita and I. Takewaki * \\ Dept. of Urban \& Environmental Eng., Graduate School of Eng., Kyoto University, \\ Kyotodaigaku-Katsura, Nishikyo, Kyoto 615-8540, Japan
}

\begin{abstract}
A stochastic model is treated of bi-directional horizontal ground motions (2DGM). It is shown that, in comparison with the Penzien-Watabe model (1975), the cross power spectral density (PSD) function between 2DGM along the building structural axes can be treated in a more general manner by using an extended Penzien-Watabe model introduced in this paper. The auto PSD functions of 2DGM along the building structural axes are assumed to be given and the cross PSD function between these 2DGM is treated as a complex unknown function. A critical excitation problem is then considered for a one-story one-span moment resisting three-dimensional frame. The corner-fiber stress at the column-end is taken as the objective function and the worst cross PSD function of the 2DGM is determined so that the maximum corner-fiber stress at the column-end is maximized. It is shown that the real part (co-spectrum) and the imaginary part (quad-spectrum) of the worst cross PSD function can be obtained by a devised algorithm including the interchange of the double maximization procedure in the time and frequency domains.
\end{abstract}

Keywords: Critical excitation, Multi-component inputs, Cross-correlation function, Coherency, Cross spectrum, Stochastic process, Space frame

\footnotetext{
* Corresponding author. Tel.: +81-75-383-3294, Fax: +81-75-383-3297, E-mail: takewaki@archi.kyoto-u.ac.jp
} 


\section{Introduction}

The ground motion is a realization in space and simultaneous consideration of multiple components of ground motion is inevitable in the reliable design of structures $[1,2]$. It is assumed practically that there exists a set of principal axes in the ground motions $[3,4]$. It is well recognized in the literature that the principal axes are functions of time and change their directions during the ground shaking. In the current structural design practice, the effect of the multi-component ground motions is often taken into account by use of the SRSS method (square root of the sum of the squares) or the CQC3 method (extended Complete Quadratic Combination rule [5]).

In the SRSS method, the maximum responses under respective ground motions are combined by the rule of SRSS. The SRSS method assumes the statistical independence among the respective ground motions. However, the multi-component ground motions have some statistical dependence.

On the other hand, the CQC3 rule is well known as a response spectrum method which can take into account the effect of correlation between the components of ground motions. Although an absolute value of a cross power spectral density (PSD) function has been described by the correlation coefficient, the CQC3 rule can not treat directly, in the sense of direct treatment of both real and imaginary parts, the cross PSD functions of multi-component ground motions. Menun and Der Kiureghian [6] and Lopez et al. [7] employed the CQC3 method as the response evaluation method and discussed the critical states, e.g. a critical loading combination or a critical incident angle. Athanatopoulou [8] investigated the effect of incident angle of ground motions on structural response without use of the Penzien-Watabe model [3] and pointed out the significance of considering multiple inputs in the practical seismic design. The approach is applicable only to a set of recorded motions. In this paper, the cross PSD function in terms of both real and imaginary parts will be discussed in more detail from the viewpoint of critical excitation.

A problem of critical excitation is considered in this paper for a one-story one-span moment resisting three-dimensional (3-D) frame subjected to bi-directional horizontal ground motions (2DGM). Because the horizontal ground motions are known to be influential to 
most of ordinary building structures, only horizontal ground motions are treated here. The two horizontal ground accelerations are modeled as nonstationary random processes whose auto PSD functions are known. A critical excitation problem is formulated such that the worst cross PSD function of the 2DGM is determined for the maximum mean-squares extreme-fiber stress of the column at the top. It is found that the real part (co-spectrum, e.g. see Nigam [9]) and the imaginary part (quad-spectrum) of the worst cross PSD function can be obtained by a devised algorithm including the interchange of the double maximization procedure in the time and frequency domains.

The critical excitation problems have been treated extensively by many researchers, e.g. Drenick [10], Shinozuka [11], Iyengar and Manohar [12], Manohar and Sarkar [13], Abbas and Manohar [14-16], Takewaki [17-22]. The works by Sarkar and Manohar [23,24], Abbas and Manohar $[15,16])$ are concerned with the present paper. Sarkar and Manohar $[23,24]$ and Abbas and Manohar [15] formulated interesting problems and solved the problems via sophisticated mathematical insights. In particular, they revealed that the critical correlation occurs under the condition of perfect coincidence of the multiple-support inputs with the corresponding transfer functions. Furthermore Abbas and Manohar [15] discussed a critical excitation problem of a stack-like structure subjected to horizontal and vertical simultaneous inputs with the reliability index as the objective function. They determined the critical PSD matrix using response surface models. The present paper formulates a similar problem for a different model (multi-component input) with different variables in the complex plane of the cross PSD function of ground motions. Especially the relationship of the building principal axes with the ground-motion principal axes produces an interesting aspect.

\section{Penzien-Watabe model and extended Penzien-Watabe model}

\subsection{Penzien-Watabe model}

The CQC3 rule is based on the Penzien-Watabe model (P-W model; [3]). The P-W model assumes the existence of the principal axes $Z_{1}$ and $Z_{2}$ along which the correlation coefficient of ground motions is zero. One principal axis in the horizontal plane is directed to the fault and the other is perpendicular to the former one. 
Although the CQC3 rule is a known method of response analysis for 2DGM, the correlation between 2DGM is fixed rigidly. In order to generalize the correlation between the 2DGM in a feasible complex plane of the cross PSD function, a new ground input model is proposed in this paper. Then, the CQC3 rule can be regarded as a special case of response evaluation using the input model proposed in this paper. This will be explained later in section 5. A brief explanation is shown in Fig.1 in the form of flow chart.

Consider a one-story one-span 3-D frame. It is assumed that two axes $X_{1}$ and $X_{2}$ are perpendicular to each other and along the building structural axes. Let $S_{Z_{1}}(\omega)$ and $S_{Z_{2}}(\omega)$ denote the auto PSD functions along the principal axes $Z_{1}, Z_{2}$ of ground motions respectively. According to the $\mathrm{P}-\mathrm{W}$ model, 2DGM along $Z_{1}, Z_{2}$ are regarded to be completely uncorrelated. The auto PSD functions of ground motions along $X_{1}, X_{2}$ are determined from the auto PSD functions of 2DGM along $Z_{1}, Z_{2}$. The auto PSD functions along $X_{1}, X_{2}$ are described by $S_{11}(\omega)$ and $S_{22}(\omega)$, respectively.

It can be shown (see Appendix 1) that the sum of $S_{Z_{1}}(\omega)$ and $S_{Z_{2}}(\omega)$ is to be equal to the sum of $S_{11}(\omega)$ and $S_{22}(\omega)$. Furthermore, the coherence function between 2DGM along $X_{1}$ and $X_{2}$ is also denoted as

$$
\rho_{12}\left(\gamma_{\text {org }}, \theta\right)=\frac{\left(1-\gamma_{\text {org }}\right) \sin 2 \theta}{\sqrt{\left(1+\gamma_{\text {org }}\right)^{2}-\left(1-\gamma_{\text {org }}\right)^{2} \cos ^{2} 2 \theta}}
$$

where $\gamma_{\text {org }}=S_{Z_{2}}(\omega) / S_{Z_{1}}(\omega) . \quad \theta$ is the angle of rotation (incident angle) between the two horizontal axes $Z_{1}, X_{1}$. Fig.2 shows the coherence function expressed by Eq.(1) with various values of $\gamma_{\text {org }}$ for varied rotation (incident) angle. In Fig.2, when $\gamma_{\text {org }}$ is zero, the coherence function $\rho_{12}$ is reduced to 1 at any $\theta$ except $\theta=0$ and $\theta=\pi / 2$. This means that the components along $X_{1}$ and $X_{2}$ have perfect correlation under uni-directional ground motion along the major principal axis of ground motion.

\subsection{Extended Penzien-Watabe model}

The P-W model is often used in the modeling of multi-component ground motions. Although the coherence function of 2DGM along $X_{1}$ and $X_{2}$ can be given in terms of $\gamma_{\text {org }}$ 
and $\theta$ as shown in Eq.(1), the cross PSD function can not be treated directly in the CQC3 rule. For that reason, it is supposed in this paper that the cross PSD function between 2DGM along $X_{1}$ and $X_{2}$ can take any value in the feasible complex plane. From the definition of the coherence function, the co-spectrum (real part of cross PSD) $C_{12}(\omega)$ and quad-spectrum (imaginary part of cross PSD) $Q_{12}(\omega)$ must satisfy the following relation.

$$
C_{12}(\omega)^{2}+Q_{12}(\omega)^{2} \leq\left\{\rho_{12}\left(\gamma_{\text {org }}, \theta\right)\right\}^{2} S_{11}(\omega) S_{22}(\omega)
$$

This model is called the extended P-W model hereafter. It may be possible to incorporate the extended P-W model into the stochastic response evaluation method. In that case, a new critical excitation problem can be constructed in which the critical cross PSD function is searched in the feasible complex plane represented by Eq.(2). This method can be regarded as an extended method of the CQC3 rule based on the P-W model.

\section{Stochastic response to 2DGM described by extended Penzien-Watabe model}

\subsection{Definition of nonstationary ground motion}

It is assumed here that the one-directional horizontal motions can be described by the following uniformly modulated nonstationary model.

$\ddot{u}_{g i}(t)=c_{i}(t) w_{i}(t) \quad(i=1,2)$

where $c_{i}(t)$ is an envelope function and $w_{i}(t)$ is a stationary random process. The envelope function is given by

$$
\begin{array}{lc}
c_{i}(t)=\left(t / t_{0}\right)^{2} & \left(0 \leq t \leq t_{0}\right) \\
c_{i}(t)=1.0 & \left(t_{0} \leq t \leq t_{1}\right) \\
c_{i}(t)=e^{-0.24\left(t-t_{1}\right)} & \left(t_{1} \leq t \leq t_{f}\right)
\end{array}
$$

The auto PSD function of $w_{i}(t)$ in Eq.(3) is assumed to be given by

$$
S_{i i}(\omega)=S_{V, h=0}^{(i)} /\left\{2 \pi T\left|c_{i}(t)\right|_{\max }\right\} \quad(i=1,2)
$$

where $T$ is the time duration and $h$ is the damping ratio. $S_{V, h=0}^{(i)}$ is the velocity response spectrum for null damping ratio. 


\subsection{Stochastic response evaluation in frequency domain}

\subsubsection{Structure model}

Consider a 3-D frame subjected to 2DGM $\ddot{u}_{g 1}, \ddot{u}_{g 2}$ along the building structural axes $X_{1}, X_{2}$. It is assumed that the center of mass is coincident with the center of stiffness and the torsional response does not occur so long as there is no rotational input. The columns have a square-tube cross section and the beams have a wide-flange cross section as shown in Fig.3. The story height is $H$ and the span length of the plane frame of interest in the first part of this section is $L_{1}$. The span length in the other direction is denoted by $L_{2}$. Let $E, I_{b}, I_{c}, Z_{c}$ denote the Young's modulus of beam and column, the second moment of area of beam, that of column and the section modulus of column, respectively. The mass on one plane frame is denoted by $m_{1}$.

Assume that each plane frame of the 3-D model can be expressed by an SDOF model. The equivalent horizontal stiffness of the SDOF model is expressed by (see Appendix 2)

$$
k_{1}=\frac{12 E I_{c}\left\{1+6\left(I_{b} / I_{c}\right) \cdot\left(H / L_{1}\right)\right\}}{H^{3}\left\{2+3\left(I_{b} / I_{c}\right) \cdot\left(H / L_{1}\right)\right\}}
$$

The extreme-fiber stress at the top of the column under one-directional horizontal motion may be expressed by (see Appendix 2)

$\sigma_{B A}^{1}(t)=\left\{6 E I_{b} /\left(Z_{c} L_{1}\right)\right\} \theta_{B}=A_{\sigma 1} u_{1}(t)$

where $A_{\sigma 1} \equiv 18 E I_{b} /\left[H L_{1} Z_{c}\left\{2+3\left(I_{b} / I_{c}\right) \cdot\left(H / L_{1}\right)\right\}\right]$.

Let $\omega_{1}=\sqrt{k_{1} / m_{1}}$ denote the fundamental natural circular frequency in the horizontal vibration of the SDOF model. The horizontal displacement of the floor can be derived as

$u_{1}(t)=\int_{0}^{t}\left\{-\ddot{u}_{g 1}(\tau)\right\} g_{1}(t-\tau) d \tau$

where $g_{1}(t)$ is the well-known unit impulse response function.

Using Eqs. (7) and (8), $\sigma_{B A}^{1}(t)$ can be expressed as

$$
\sigma_{B A}^{1}(t)=A_{\sigma 1} \int_{0}^{t}\left\{-\ddot{u}_{g 1}(\tau)\right\} g_{1}(t-\tau) d \tau
$$


Let $\sigma_{B A}^{2}(t)$ denote the extreme-fiber stress at the top of the column under another horizontal motion $\ddot{u}_{g 2}$. The same equations as those in the direction $X_{1}$ can be used only by replacing $L_{1}$ by $L_{2}$ and other parameters in the direction $X_{1}$ by those in the direction $X_{2}$. The sum of the extreme-fiber stresses at the top of the column under 2DGM may be expressed by

$$
f(t)=\sigma_{B A}^{1}(t)+\sigma_{B A}^{2}(t)
$$

\subsubsection{Stochastic response evaluation in frequency domain}

The auto-correlation function of $f(t)$ defined in Eq.(10) can be expressed by

$$
\begin{aligned}
E\left[f\left(t_{1}\right) f\left(t_{2}\right)\right]=E\left[\sigma_{B A}^{1}\left(t_{1}\right) \sigma_{B A}^{1}\left(t_{2}\right)\right]+E\left[\sigma_{B A}^{1}\left(t_{1}\right) \sigma_{B A}^{2}\left(t_{2}\right)\right] \\
+E\left[\sigma_{B A}^{2}\left(t_{1}\right) \sigma_{B A}^{1}\left(t_{2}\right)\right]+E\left[\sigma_{B A}^{2}\left(t_{1}\right) \sigma_{B A}^{2}\left(t_{2}\right)\right]
\end{aligned}
$$

where $E[\cdot]$ denotes the ensemble mean. The mean-squares extreme-fiber stresses in directions $X_{1}$ and $X_{2}$ derived from Eq.(11) may be expressed by (See Appendix 3)

$$
\begin{aligned}
& E\left[\sigma_{B A}^{1}(t)^{2}\right]=A_{\sigma 1}^{2} \int_{-\infty}^{\infty}\left\{B_{C}(t ; \omega)^{2}+B_{s}(t ; \omega)^{2}\right\} S_{11}(\omega) d \omega \\
& E\left[\sigma_{B A}^{2}(t)^{2}\right]=A_{\sigma 2}{ }^{2} \int_{-\infty}^{\infty}\left\{C_{c}(t ; \omega)^{2}+C_{s}(t ; \omega)^{2}\right\} S_{22}(\omega) d \omega
\end{aligned}
$$

$B_{c}(t ; \omega), B_{s}(t ; \omega), C_{c}(t ; \omega), C_{s}(t ; \omega)$ are defined in Appendix 3.

The cross terms in Eq.(11) can be transformed into (See Appendix 4)

$$
\begin{aligned}
& E\left[\sigma_{B A}^{1}(t) \sigma_{B A}^{2}(t)\right]+E\left[\sigma_{B A}^{2}(t) \sigma_{B A}^{1}(t)\right] \\
& =2 A_{\sigma 1} A_{\sigma 2} \int_{-\infty}^{\infty}\left\{f_{1}(t ; \omega) C_{12}(\omega)+f_{2}(t ; \omega) Q_{12}(\omega)\right\} d \omega
\end{aligned}
$$

where $C_{12}$ and $Q_{12}$ are the co-spectrum and quad-spectrum of the cross PSD function and

$$
\begin{aligned}
& f_{1}(t ; \omega)=B_{c}(t ; \omega) C_{c}(t ; \omega)+B_{S}(t ; \omega) C_{s}(t ; \omega) \\
& f_{2}(t ; \omega)=B_{c}(t ; \omega) C_{s}(t ; \omega)-B_{s}(t ; \omega) C_{c}(t ; \omega)
\end{aligned}
$$

Finally the mean-squares of the sum of extreme-fiber stresses at the top of the column under 2DGM may be expressed by 


$$
\begin{aligned}
& E\left[\left\{\sigma_{B A}^{1}(t)+\sigma_{B A}^{2}(t)\right\}^{2}\right] \\
& =A_{\sigma 1}^{2} \int_{-\infty}^{\infty}\left\{B_{c}(t ; \omega)^{2}+B_{s}(t ; \omega)^{2}\right\} S_{11}(\omega) d \omega \\
& \quad+2 A_{\sigma 1} A_{\sigma 2} \int_{-\infty}^{\infty}\left\{f_{1}(t ; \omega) C_{12}(\omega)+f_{2}(t ; \omega) Q_{12}(\omega)\right\} d \omega \\
& \quad+A_{\sigma 2}{ }^{2} \int_{-\infty}^{\infty}\left\{C_{c}(t ; \omega)^{2}+C_{s}(t ; \omega)^{2}\right\} S_{22}(\omega) d \omega
\end{aligned}
$$

\section{Critical excitation method for worst cross PSD function between 2DGM}

The critical excitation problem may be stated as: Find the cross PSD function $S_{12}(\omega)=C_{12}(\omega)+\mathrm{i} Q_{12}(\omega)$ of 2DGM so as to achieve $\max _{S_{12}(\omega)} \max _{t} E\left[\left\{\sigma_{B A}^{1}(t)+\sigma_{B A}^{2}(t)\right\}^{2}\right]$.

When the time $t$ is fixed and the frequency $\omega$ is specified, the transfer functions $f_{1}(t ; \omega)$ and $f_{2}(t ; \omega)$ defined in Eqs.(15a, b) can be regarded as coefficients, not functions of $t$ and $\omega$. Therefore the integrand in the second term of Eq.(16) can be regarded as the function $z\left(C_{12}, Q_{12}\right)$ of $C_{12}$ and $Q_{12}$.

$$
z\left(C_{12}, Q_{12}\right)=f_{1}(t ; \omega) C_{12}(\omega)+f_{2}(t ; \omega) Q_{12}(\omega)
$$

Fig.4 illustrates the structure of the critical excitation problem. The critical excitation problem is to maximize

$$
z^{*}\left(C_{12}, Q_{12}\right)=2 A_{\sigma 1} A_{\sigma 2}\left\{f_{1}(t ; \omega) C_{12}(\omega)+f_{2}(t ; \omega) Q_{12}(\omega)\right\}
$$

under the constraint (2). The critical co-spectrum and quad-spectrum can then be obtained analytically as

$$
\begin{aligned}
& C_{12}(\omega)=\rho_{12}\left(\gamma_{\text {org }}, \theta\right) f_{1}(t ; \omega) \frac{\sqrt{S_{22}(\omega) S_{11}(\omega)}}{\sqrt{f_{1}(t ; \omega)^{2}+f_{2}(t ; \omega)^{2}}} \\
& Q_{12}(\omega)=\rho_{12}\left(\gamma_{\text {org }}, \theta\right) f_{2}(t ; \omega) \frac{\sqrt{S_{22}(\omega) S_{11}(\omega)}}{\sqrt{f_{1}(t ; \omega)^{2}+f_{2}(t ; \omega)^{2}}}
\end{aligned}
$$

It should be noted that Eqs.(19a,b) include the coherence function $\rho_{12}\left(\gamma_{\text {org }}, \theta\right)$ and are different from the equations derived in Reference [25]. Abbas and Manohar [15] had obtained a similar result for a different problem of multiple inputs.

Fig.5 indicates the solution algorithm. Substitution of Eqs.(19a, b) into Eq.(14) leads 
to the expression of the cross term.

$$
\begin{aligned}
& E\left[\sigma_{B A}^{1}(t) \sigma_{B A}^{2}(t)\right]+E\left[\sigma_{B A}^{2}(t) \sigma_{B A}^{1}(t)\right] \\
& =2 \rho_{12}\left(\gamma_{\text {org }}, \theta\right) A_{\sigma 1} A_{\sigma 2} \int_{-\infty}^{\infty} \sqrt{f_{1}(t ; \omega)^{2}+f_{2}(t ; \omega)^{2}} \sqrt{S_{22}(\omega) S_{11}(\omega)} d \omega
\end{aligned}
$$

\section{Numerical Example}

\subsection{Response to 2DGM with the constraint of sum of auto PSD functions}

In most of the current structural design practice, safety and functionality checks are made with respect to one-directional earthquake input. This is because the ground motion model for multi-component inputs is complicated and a well-accepted model of practical use has never been presented except a few (e.g. Eurocode, IBC International Code). In addition, it may be understood that an approximate safety margin is incorporated in the magnitude of one-directional input. In this section, the effect of bi-directional input on the seismic response is investigated through the comparison with the response by CQC3 rule (perfectly correlated; although CQC3 does not correspond to the perfectly correlated case, this terminology is used symbolically) or SRSS rule (uncorrelated). The effect of correlation of 2DGM on the response is also clarified. Fig.6 shows the flow chart of the aim in this section and the relationship with section 5.2. The given structural parameters are shown in Tables 1 and 2.

Consider the case where the auto PSD function ratio in two directions $X_{1}, X_{2}$ is varied under the condition that the sum of the auto PSD functions in two directions is constant. This is because the intensity of the uni-directional input as the combined component of two-directional input should be regarded to be constant. For uni-directional input $\left(\gamma_{\text {org }}=0\right)$ along the major principal axis of ground motion, the coherence function between the ground motions along the building structural axes is fixed to 1.0 (See Eq. (1)). The auto PSD function ratios along the building structural axes are chosen as $\gamma=S_{22} / S_{11}=0,0.25,0.75$ and 1.0. The 2DGM along the building structural axes with the auto PSD function ratio of 1.0 and coherence $=1.0$ coincides with the uni-directional input along the major ground principal 
axis of $\theta=\pi / 4$. The common envelope function $c_{1}(t), c_{2}(t)$ is shown in Fig.7. The parameters in Eq.(4) are taken as $c_{0}=3(\mathrm{~s}), c_{1}=12.5(\mathrm{~s})$ and $c_{f}=40.0$ (s) here. Figs.8(a)-(c) indicate the auto PSD functions of $w_{1}(t), w_{2}(t)$ for various $\gamma$ with the constraint of sum of auto PSD functions. The simulated ground acceleration using this PSD functions has the maximum value of about 1G. The span length $L_{2}$ is specified as $15(\mathrm{~m})$ and the span length $L_{1}$ has been varied continuously from 10(m) to 30(m).

Fig.9 shows the comparison of the response to critically correlated 2DGM along the building structural axes with the response to uncorrelated bi-directional input. The curve indicated as 'uncorrelated' corresponds to the SRSS response and the curve indicated as 'critically correlated' presents the critical response derived in this paper. In addition, the responses to 2DGM which have fixed correlation functions, i.e. $C_{12}=\sqrt{S_{11} S_{22}}$ and $Q_{12}=0$ ("Perfectly correlated" without phase delay), $C_{12}=Q_{12}=\sqrt{S_{11} S_{22}} / \sqrt{2}$ (case 2), $C_{12}=0$ and $Q_{12}=\sqrt{S_{11} S_{22}}$ (case 3 ), are also plotted. It can be seen that the critical response and the response to the input model with $C_{12}=\sqrt{S_{11} S_{22}}$ and $Q_{12}=0$ almost coincide in the model with the span of $L_{1}=15(\mathrm{~m})$.

It can be observed from Fig.9 that the critical response is amplified around $L_{1}=15(\mathrm{~m})$ where the lengths of span in two directions are equal and the natural frequencies of the model in two directions are equal. It can also be observed that, as the span length $L_{1}$ becomes longer than 15(m), the critical response for the input model of $\gamma=0.25$ becomes larger than those for $\gamma=0.75,1.0$. This is because, as the span becomes longer, a horizontal stiffness along the long span decreases. It can be concluded that the critical incident angle of multi-component ground motions may exist depending on the combination of structural stiffnesses due to difference in span lengths.

Fig.10 shows the increase ratio of the critical response to 2DGM of various auto PSD function ratios from the SRSS response. In this case, the increased ratio is about $40 \%$ at $L_{1}=15(\mathrm{~m})$. This implies that most of the present design code using only one-directional input $(\gamma=0)$ for safety check are not sufficient for extreme loading.

Fig.11 shows the co-spectrum and quad-spectrum of the critical cross PSD function for $\gamma=1$ and $L_{1}=25(\mathrm{~m})$. 
5.2 Response to 2DGM described by extended Penzien-Watabe model: analysis from the viewpoint of critical incident angle

Since the analytical solution has been obtained as Eqs.19(a, b), the critical incident angle can be searched parametrically in an efficient manner for which the response quantity can be maximized for each combination of span length. The right figure in Fig.6 shows the flow chart indicating the aim in this section. While the auto PSD function ratio along the building structural axes has been treated directly in section 5.1, the auto PSD functions along the principal axes of ground motions are treated directly in this section. In other words, the physical meaning of ground motions is taken into account in detail in this section.

Consider the case where the ratio $\gamma_{\text {org }}$ of the auto PSD functions along the principal axes of ground motions is assumed to be fixed to $0.0,0.25,0.75,1.0$ and the angle between the two sets of axes is varied continuously from $0(\mathrm{rad})$ to $\pi / 2$ (rad). The structural plan is given as $L_{1}=15(\mathrm{~m}), L_{2}=25(\mathrm{~m})$.

Figs.12(a)-(d) show the comparison of the critical response with the corresponding SRSS response in the case of four ratios $\gamma_{\text {org }}$ under various incident angles. The auto PSD functions along the building structural axes are determined from those along the principal axes of ground motions in terms of $\theta$ and $\gamma_{\text {org }}$ (See Appendix 1). Since the case of $\gamma_{\text {org }}=0.0$ shown in Fig.12(a) can be regarded as the uni-directional input along the ground major principal axis, some cases in this figure have already been shown in Figs.9(a)-(d). Figs.9(a)-(d) at span $=25(\mathrm{~m})$ correspond to Fig.12(a) at $\theta=0[\mathrm{rad}], \quad \theta=0.078 \pi[\mathrm{rad}]$ $\left(=14.0^{\circ}\right) \quad, \quad \theta=0.20 \pi[\mathrm{rad}]\left(=36.9^{\circ}\right) \quad$ and $\quad \theta=\pi / 4[\mathrm{rad}]\left(=45^{\circ}\right), \quad$ respectively. $\quad$ From Figs.12(a)-(c), it can be understood that there exists a critical incident angle which maximizes the response quantity by considering the effect of critical correlation between 2DGM along the building structural axes. It should be noted that, while Lopez et al (2000) used the P-W model, the present paper introduced the extended P-W model and took into account the critical cross PSD function between 2DGM.

The critical response and the corresponding SRSS response have the same value at $\theta=0.0$ and $\theta=\pi / 2$ in Figs.12(a)-(c). This is because, in the case of $\gamma_{\text {org }}=0.0$ shown 
in Fig.12(a), there is no component of ground motion along the other building structural axis at $\theta=0.0$ or $\theta=\pi / 2$ and the effect of the correlation of the 2DGM does not exist. While in the case of $\gamma_{\text {org }} \neq 0.0$, i.e. in Figs.12(b), (c), the coherence function $\rho_{12}\left(\gamma_{\text {org }}, \theta\right)$ based on the P-W model is 0.0 at $\theta=0.0$ or $\theta=\pi / 2$ and the cross term of Eq.(11) does not exist. In Fig.12(d), there is no differences between two lines. This is because 2DGM along the building structural axes are uncorrelated due to $\rho_{12}\left(\gamma_{\text {org }}, \theta\right)=0.0$ for $\gamma_{\text {org }}=1.0$. Comparing Fig.12(a) with Figs.12(b)-(d), it can be observed that the maximum value, shown in Figs.12(b)-(d), of the response to the 2DGM along the principal axes of ground motions does not exceed that to the uni-directional input shown in Fig.12(a). This may result from the fact that (1) the coherence is 1.0 in Fig.12(a) and is smaller than 1.0 in Figs.12(b)-(d) and (2) the concentrated uni-directional input is more effective in maximizing the extreme-fiber stress.

Under the constraint of sum of auto PSD functions along the principal axes of ground motions, it may be concluded that the response evaluation to the uni-directional input along the principal axes of ground motions $\left(\gamma_{\text {org }}=0.0\right.$ ) is sufficient as far as the maximum value of response quantity is concerned.

\subsection{Comparison of response to critically correlated 2DGM with that to perfectly correlated} $2 D G M$

In order to understand the property of the critically correlated ground motions more deeply, the comparison with the perfectly correlated ground motions without time delay has been made. The structural plan is given as $L_{1}=15(\mathrm{~m}), L_{2}=25(\mathrm{~m})$. Fig.13(a) shows two horizontal ground motions with the critical correlation for input model of $\gamma_{\text {org }}=0$ and $\theta=0.106 \pi[\mathrm{rad}]\left(=19.0^{\circ}\right)$ (critical incident angle shown in Fig.12(a)). This set has been generated by using random numbers. On the other hand, Fig.13(b) indicates two horizontal ground motions with the perfect correlation without time delay for $\gamma_{\text {org }}=0$ and $\theta=0.106 \pi[\operatorname{rad}]\left(=19.0^{\circ}\right)$. Fig.14 illustrates the root-mean-square of column-end extreme-fiber stress to these two sets of horizontal ground motions. It can be observed that the response to the critically correlated ground motions could become about 1.5 times larger 
than that to the perfectly correlated ground motions without time delay.

\subsection{Analysis of recorded 2DGM}

The correlation between recorded 2DGM should be compared with the result of the critical excitation method developed in this paper. In this section, the coherence function between the recorded 2DGM (El Centro NS and EW during Imperial Valley 1940, SCT1 NS and EW during Mexico Michoacan 1985) is calculated.

The auto PSD functions and cross PSD functions have been calculated from the Fourier transforms by using the Welch-Bartlett's method. The starting time of the window with the duration $T$ (5s in El Centro and 10s in SCT1) was changed successively (time-lag of 0.02s) and the corresponding set of data for the 100 windows was chosen to represent candidates of the ensemble mean. Then the procedure of ensemble mean was taken of the functions computed from the Fourier transforms.

Fig.15(a) shows the representative acceleration records of El Centro NS and EW and Fig.15(b) illustrates the cross PSD function of both motions. For these data, Fig.15(c) indicates the coherence function. It has been understood from several parametric analyses that the coherence function is affected significantly by the portion of ground motions. On the other hand, Figs.16(a)-(c) illustrate the corresponding ones for SCT1 NS and EW. It can be seen that the cross PSD function of SCT1 NS and EW has a peculiar characteristic due to the predominant period of these motions. In Fig.16(c), the cases of the numbers 200 and 300 of windows have also been examined in using the Welch-Bartlett's method. It can be observed that the coherence strongly depends on the type of earthquake ground motions. Furthermore, as stated, the coherence also depends on the portion of ground motions (this data are not shown here due to page limit). The prediction of the coherence function before its occurrence is quite difficult and the critical excitation method will provide a meaningful insight even in these circumstances.

As for the reality of critical excitation methods, a severe ground motion attacked recently (July 16, 2007) the city of Kashiwazaki, Niigata Prefecture in Japan and many old wood houses were destroyed. It has been reported that a peculiar ground motion as shown in 
Fig.17(a) has been observed and the ground motion had a predominant period of 2.5 (s). This period is thought to be resonant with the natural period of old wood houses with heavy roofs. This ground motion is very similar to one, shown in Fig.17(b), predicted in Reference (Takewaki 2004a). It should be noted that a large nuclear reactor facility is located in the city of Kashiwazaki and that facility had relatively minor damage. Further damage investigation is being conducted even now. This ground motion strongly supports the importance of introducing the critical excitation methods especially for important structures.

\section{Conclusions}

An extended Penzien-Watabe model has been proposed in which the cross PSD function of 2DGM can be treated in a more relaxed manner. While only the coherence function, i.e. the absolute value of the cross PSD function, can be treated in the P-W model, the direct treatment of the cross PSD function has been made possible in the extended P-W model. The following conclusions have been derived.

(1) A critical excitation problem has been formulated for a one-story one-span moment resisting 3-D frame subjected to the 2DGM obeying the proposed extended P-W model. The objective function is the corner-fiber stress at the column-end. The extended P-W model is an extended version of the P-W model including an additional information on the cross PSD function as a complex function.

(2) The mean-squares corner-fiber stress at the column-end has been shown to be the sum of the term due to the 2DGM and that due to their correlation. Since the auto PSD functions of 2DGM are given and prescribed, the maximization in the critical excitation problem means the maximization of the correlation term of 2DGM.

(3) The real part (co-spectrum) and the imaginary part (quad-spectrum) of the worst cross PSD function can be obtained by a devised algorithm including the interchange of the double maximization procedure in the time and cross PSD function domains.

(4) Numerical examples indicate that the proposed algorithm can work very well. The root-mean-square corner-fiber stress at the column-end to the critical combination of the 2DGM becomes more than ten percent larger than that by the SRSS estimate of 
corner-fiber stress at the column-end due to the 2DGM. When the horizontal stiffnesses along the building structural axes coincide with each other, the response to the critical excitation becomes about forty $(\simeq \sqrt{2})$ percent larger than that by the SRSS estimate.

(5) Analytical solutions, Eqs.(19a, b), have enabled the efficient parametric analysis of critical incident angle (see Fig.12).

(6) The coherence function between the 2DGM of recorded earthquakes has been calculated. The coherence strongly depends on the type of earthquake ground motions and the prediction of the coherence function before its occurrence is quite difficult. The critical excitation method will provide a meaningful insight even in these circumstances.

\section{Acknowledgements}

Part of the present work is supported by the Grant-in-Aid for Scientific Research of Japan Society for the Promotion of Science (No.18360264, 21360267). This support is greatly appreciated. The ground motion record at Kashiwazaki site was provided by the K-net supported by Japanese Government.

\section{Appendix 1: Computation of coherence function and transformation of PSD matrices}

Let $\ddot{u}_{g 1}$ and $\ddot{u}_{g 2}$ denote the ground-motion accelerations along the building structural axes $X_{1}$ and $X_{2}$, respectively. Under the 2DGM along the principal axes of ground motions in the P-W model, $\ddot{u}_{g 1}$ and $\ddot{u}_{g 2}$ are described by

$$
\left\{\begin{array}{l}
\ddot{u}_{g 1} \\
\ddot{u}_{g 2}
\end{array}\right\}=\left[\begin{array}{cc}
\cos \theta & \sin \theta \\
-\sin \theta & \cos \theta
\end{array}\right]\left\{\begin{array}{l}
\ddot{u}_{z 1} \\
\ddot{u}_{z 2}
\end{array}\right\}
$$

where $\ddot{u}_{z 1}$ and $\ddot{u}_{z 2}$ are the ground-motion accelerations along the principal axes of ground motions. $\theta$ denotes the angle between two sets of horizontal axes (=incident angle).

Let $\mathbf{S}_{\ddot{Z} Z}(\omega)$ denote the auto PSD matrix of the components along the principal axes of ground motions. Then the PSD matrix, consisting of $S_{11}, S_{22}, S_{12}, S_{21}$, of the components along the building structural axes may be described as 


$$
\begin{aligned}
& \mathbf{S}_{\ddot{X} \ddot{X}}(\omega)=\left[\begin{array}{cc}
\cos \theta & \sin \theta \\
-\sin \theta & \cos \theta
\end{array}\right] \mathbf{S}_{\ddot{Z} \ddot{Z}}(\omega)\left[\begin{array}{cc}
\cos \theta & -\sin \theta \\
\sin \theta & \cos \theta
\end{array}\right] \\
& \mathbf{S}_{\ddot{Z} \ddot{Z}}(\omega)=\left[\begin{array}{cc}
S_{\ddot{Z}_{1} \ddot{Z}_{1}}(\omega) & 0 \\
0 & S_{\ddot{Z}_{2} \ddot{Z}_{2}}(\omega)
\end{array}\right]
\end{aligned}
$$

The coherence function between the components of ground motions along the building structural axes is defined by

$$
\rho_{12}=\frac{E\left[\ddot{u}_{g 1} \ddot{u}_{g 2}\right]}{\sqrt{E\left[\ddot{u}_{g 1}^{2}\right] E\left[\ddot{u}_{g 2}^{2}\right]}}
$$

where $E[\cdot]$ denotes the ensemble mean. It is assumed in the P-W model that there is no correlation between the 2DGM along the principal axes of ground motions (i.e. $\left.E\left[\ddot{u}_{z 1} \ddot{u}_{z 2}\right]=0\right)$. Let $\gamma_{\text {org }}$ denote the ratio of the auto PSD functions $S_{\ddot{Z}_{2} \ddot{Z}_{2}}(\omega) / S_{\ddot{z}_{1} \ddot{z}_{1}}(\omega)$ along the principal axes of ground motions. Substitution of $\ddot{u}_{g 1}$ and $\ddot{u}_{g 2}$ in Eq.(A1) into Eq.(A4) and some manipulations provide

$$
\rho_{12}\left(\gamma_{\text {org }}, \theta\right)=\frac{\left(1-\gamma_{\text {org }}\right) \sin 2 \theta}{\sqrt{\left(1+\gamma_{\text {org }}\right)^{2}-\left(1-\gamma_{\text {org }}\right)^{2} \cos ^{2} 2 \theta}}
$$

\section{Appendix 2: Horizontal stiffness of frame}

Let $u_{1}$ and $\phi_{A B}$ denote the horizontal displacement of the upper node in the frame and the angle of member rotation of column, respectively. When the horizontal force is denoted by $P_{1}$, the horizontal stiffness of the plane frame can be expressed as

$$
k_{1}=P_{1} / u_{1}=P_{1} /\left(H \cdot \phi_{A B}\right)
$$

The extreme-fiber stress at the top of the column under one-directional horizontal input may be expressed by

$$
\sigma_{B A}^{1}(t)=\left\{6 E I_{b} /\left(Z_{c} L_{1}\right)\right\} \theta_{B}
$$

From the moment equilibrium around the node $\mathrm{B}$, the angle of rotation of the node $\mathrm{B}$ can be expressed by

$$
\theta_{B}=3 \phi_{A B} /\left[2+3\left(I_{b} / I_{c}\right) \cdot\left(H / L_{1}\right)\right]
$$


Eq.(A8) and the equation of story equilibrium provide

$$
\phi_{A B}=\frac{P_{1} H^{2}\left\{2+3\left(I_{b} / I_{c}\right) \cdot\left(H / L_{1}\right)\right\}}{12 E I_{c}\left\{1+6\left(I_{b} / I_{c}\right) \cdot\left(H / L_{1}\right)\right\}}
$$

Then the story stiffness can be expressed by

$$
k_{1}=\frac{12 E I_{c}\left\{1+6\left(I_{b} / I_{c}\right) \cdot\left(H / L_{1}\right)\right\}}{H^{3}\left\{2+3\left(I_{b} / I_{c}\right) \cdot\left(H / L_{1}\right)\right\}}
$$

\section{Appendix 3: Stochastic response 1}

The auto-correlation function of $\sigma_{B A}^{1}(t)$ can be expressed by

$$
\begin{aligned}
& E\left[\sigma_{B A}^{1}\left(t_{1}\right) \sigma_{B A}^{1}\left(t_{2}\right)\right] \\
& =A_{\sigma 1}^{2} \int_{0}^{t_{1}} \int_{0}^{t_{2}}\left[c_{1}\left(\tau_{1}\right) c_{1}\left(\tau_{2}\right) g_{1}\left(t_{1}-\tau_{1}\right) g_{1}\left(t_{2}-\tau_{2}\right) E\left[w_{1}\left(\tau_{1}\right) w_{1}\left(\tau_{2}\right)\right]\right] d \tau_{1} d \tau_{2}
\end{aligned}
$$

The auto-correlation function of $w_{1}(t)$ can be described in terms of the auto PSD function $S_{11}(\omega)$ by

$$
E\left[w_{1}\left(\tau_{1}\right) w_{1}\left(\tau_{2}\right)\right]=\int_{-\infty}^{\infty} S_{11}(\omega) e^{\mathrm{i} \omega\left(\tau_{1}-\tau_{2}\right)} d \omega
$$

Eq.(A11) can then be modified to

$$
\begin{aligned}
& E\left[\sigma_{B A}^{1}\left(t_{1}\right) \sigma_{B A}^{1}\left(t_{2}\right)\right] \\
& =\int_{-\infty}^{\infty}\left[\begin{array}{l}
A_{\sigma 1} \int_{0}^{t_{1}} c_{1}\left(\tau_{1}\right) g_{1}\left(t_{1}-\tau_{1}\right)\left(\cos \omega \tau_{1}+\mathrm{i} \sin \omega \tau_{1}\right) d \tau_{1} \\
\times A_{\sigma 1} \int_{0}^{t_{2}} c_{1}\left(\tau_{2}\right) g_{1}\left(t_{2}-\tau_{2}\right)\left(\cos \omega \tau_{2}-\mathrm{i} \sin \omega \tau_{2}\right) d \tau_{2}
\end{array}\right] S_{11}(\omega) d \omega
\end{aligned}
$$

By substituting $t_{1}=t_{2}=t, \tau_{1}=\tau_{2}=\tau$ in Eq.(A13), the mean-squares $E\left[\sigma_{B A}^{1}(t)^{2}\right]$ can be derived as

$$
E\left[\sigma_{B A}^{1}(t)^{2}\right]=A_{\sigma 1}^{2} \int_{-\infty}^{\infty}\left\{B_{C}(t ; \omega)^{2}+B_{S}(t ; \omega)^{2}\right\} S_{11}(\omega) d \omega
$$

where

$$
B_{c}(t ; \omega) \equiv \int_{0}^{t} c_{1}(\tau) g_{1}(t-\tau) \cos \omega \tau d \tau
$$


$B_{s}(t ; \omega) \equiv \int_{0}^{t} c_{1}(\tau) g_{1}(t-\tau) \sin \omega \tau d \tau$

On the other hand, the component in the direction $X_{2}$ may be transformed into

$E\left[\sigma_{B A}^{2}\left(t_{1}\right) \sigma_{B A}^{2}\left(t_{2}\right)\right]$

$=A_{\sigma 2}^{2} \int_{0}^{t_{1}} \int_{0}^{t_{2}}\left[c_{2}\left(\tau_{1}\right) c_{2}\left(\tau_{2}\right) g_{2}\left(t_{1}-\tau_{1}\right) g_{2}\left(t_{2}-\tau_{2}\right) E\left[w_{2}\left(\tau_{1}\right) w_{2}\left(\tau_{2}\right)\right]\right] d \tau_{1} d \tau_{2}$

The auto-correlation function of $w_{2}(t)$ can be described in terms of the auto PSD function $S_{22}(\omega)$ by

$E\left[w_{2}\left(\tau_{1}\right) w_{2}\left(\tau_{2}\right)\right]=\int_{-\infty}^{\infty} S_{22}(\omega) e^{\mathrm{i} \omega\left(\tau_{1}-\tau_{2}\right)} d \omega$

The mean-squares $E\left[\sigma_{B A}^{2}(t)^{2}\right]$ can be derived as

$E\left[\sigma_{B A}^{2}(t)^{2}\right]=A_{\sigma 2}^{2} \int_{-\infty}^{\infty}\left\{C_{c}(t ; \omega)^{2}+C_{s}(t ; \omega)^{2}\right\} S_{22}(\omega) d \omega$

where

$C_{c}(t ; \omega) \equiv \int_{0}^{t} c_{2}(\tau) g_{2}(t-\tau) \cos \omega \tau d \tau$

$C_{s}(t ; \omega) \equiv \int_{0}^{t} C_{2}(\tau) g_{2}(t-\tau) \sin \omega \tau d \tau$

\section{Appendix 4: Stochastic response 2}

The cross-correlation function of $\sigma_{B A}^{1}(t)$ and $\sigma_{B A}^{2}(t)$ can be expressed as

$$
\begin{aligned}
& E\left[\sigma_{B A}^{1}\left(t_{1}\right) \sigma_{B A}^{2}\left(t_{2}\right)\right] \\
& =A_{\sigma 1} A_{\sigma 2} \int_{0}^{t_{1}} \int_{0}^{t_{2}}\left[c_{1}\left(\tau_{1}\right) c_{2}\left(\tau_{2}\right) g_{1}\left(t_{1}-\tau_{1}\right) g_{2}\left(t_{2}-\tau_{2}\right) E\left[w_{1}\left(\tau_{1}\right) w_{2}\left(\tau_{2}\right)\right]\right] d \tau_{1} d \tau_{2}
\end{aligned}
$$

The cross-correlation function of $w_{1}(t)$ and $w_{2}(t)$ can be described in terms of the cross PSD function $S_{12}(\omega)$ by

$$
E\left[w_{1}\left(\tau_{1}\right) w_{2}\left(\tau_{2}\right)\right]=\int_{-\infty}^{\infty} S_{12}(\omega) e^{\mathrm{i} \omega\left(\tau_{1}-\tau_{2}\right)} d \omega
$$

Let us introduce the definition of the cross PSD function $S_{12}(\omega)=C_{12}(\omega)+\mathrm{i} Q_{12}(\omega)$. Then Eq.(A21) can be expressed by 


$$
E\left[w_{1}\left(\tau_{1}\right) w_{2}\left(\tau_{2}\right)\right]=\int_{-\infty}^{\infty}\left\{C_{12}(\omega)+\mathrm{i} Q_{12}(\omega)\right\} e^{\mathrm{i} \omega\left(\tau_{1}-\tau_{2}\right)} d \omega
$$

The cross term can be modified into

$$
\begin{aligned}
& E\left[\sigma_{B A}^{1}(t) \sigma_{B A}^{2}(t)\right] \\
& =A_{\sigma 1} A_{\sigma 2} \int_{-\infty}^{\infty}\left[\begin{array}{l}
\int_{0}^{t} c_{1}(\tau) g_{1}(t-\tau)(\cos \omega \tau+\mathrm{i} \sin \omega \tau) d \tau \\
\times \int_{0}^{t} c_{2}(\tau) g_{2}(t-\tau)(\cos \omega \tau-\mathrm{i} \sin \omega \tau) d \tau\left\{C_{12}(\omega)+\mathrm{i} Q_{12}(\omega)\right\}
\end{array}\right] d \omega \\
& =A_{\sigma 1} A_{\sigma 2} \int_{-\infty}^{\infty}\left[\begin{array}{l}
\left.\left\{B_{c}(t ; \omega)+\mathrm{i} B_{s}(t ; \omega)\right\}\left\{C_{c}(t ; \omega)-\mathrm{i} C_{s}(t ; \omega)\right\}\left\{C_{12}(\omega)+\mathrm{i} Q_{12}(\omega)\right\}\right] d \omega
\end{array}\right.
\end{aligned}
$$

Another cross-correlation function $E\left[\sigma_{B A}^{2}\left(t_{1}\right) \sigma_{B A}^{1}\left(t_{2}\right)\right]$ may be described by

$$
\begin{aligned}
& E\left[\sigma_{B A}^{2}(t) \sigma_{B A}^{1}(t)\right] \\
& =A_{\sigma 2} A_{\sigma 1} \int_{-\infty}^{\infty}\left[\left\{C_{c}(t ; \omega)+\mathrm{i} C_{s}(t ; \omega)\right\}\left\{B_{c}(t ; \omega)-\mathrm{i} B_{s}(t ; \omega)\right\}\left\{C_{12}(\omega)-\mathrm{i} Q_{12}(\omega)\right\}\right] d \omega
\end{aligned}
$$

By combining both cross terms, the corresponding term can be expressed finally by

$$
\begin{aligned}
& E\left[\sigma_{B A}^{1}(t) \sigma_{B A}^{2}(t)\right]+E\left[\sigma_{B A}^{2}(t) \sigma_{B A}^{1}(t)\right] \\
& =2 A_{\sigma 1} A_{\sigma 2} \operatorname{Re}\left[\int_{-\infty}^{\infty}\left[\left\{B_{c}(t ; \omega)+\mathrm{i} B_{s}(t ; \omega)\right\}\left\{C_{c}(t ; \omega)-\mathrm{i} C_{s}(t ; \omega)\right\}\left\{C_{12}(\omega)+\mathrm{i} Q_{12}(\omega)\right\}\right] d \omega\right] \\
& =2 A_{\sigma 1} A_{\sigma 2} \int_{-\infty}^{\infty}\left\{f_{1}(t ; \omega) C_{12}(\omega)+f_{2}(t ; \omega) Q_{12}(\omega)\right\} d \omega
\end{aligned}
$$

\section{References}

[1] Rigato AB, Medina RA. Influence of angle of incidence on seismic demands for inelastic single-storey structures subjected to bi-directional ground motions. Eng Struct 2007; 29(10): 2593-2601.

[2] Ghersi A, Rossi PP. Influence of bi-directional ground motions on the inelastic response of one-storey in-plan irregular systems. Eng Struct 2001; 23(6): 579-591.

[3] Penzien J, Watabe M. Characteristics of 3-dimensional earthquake ground motion. Earthq Eng Struct Dyn 1975; 3: 365-374.

[4] Clough RW, Penzien J. Dynamics of Structures, second edition, Prentice Hall; 1993

[5] Smeby W, Der Kiureghian. A. Modal combination rules for multicomponent earthquake excitation. Earthq Eng Struct Dyn 1985; 13: 1-12. 
[6] Menun C, Der Kiureghian A. A replacement for the 30\%, 40\%, and SRSS rules for multicomponent seismic analysis. Earthq Spectra 1998; 14(1): 153-163.

[7] Lopez OA, Chopra AK, Hernandez JJ. Critical response of structures to multicomponent earthquake excitation Earthq Eng Struct Dyn 2000; 29: 1759-1778.

[8] Athanatopoulou AM. Critical orientation of three correlated seismic components. Eng Struct 2005; 27: 301-312.

[9] Nigam NC. Introduction to Random Vibrations 1983; MIT Press, London.

[10] Drenick RF. Model-free design of aseismic structures. J Engrg Mech Div 1970; ASCE: 96(EM4), 483-493.

[11] Shinozuka M. Maximum structural response to seismic excitations. J Engrg Mech Div 1970; ASCE: 96(EM5), 729-738.

[12] Iyengar RN, Manohar CS. Nonstationary random critical seismic excitations. J Engrg Mech 1987; ASCE: 113(4), 529-541.

[13] Manohar CS, Sarkar A. Critical earthquake input power spectral density function models for engineering structures. Earthq Eng Struct Dyn 1995; 24: 1549-1566.

[14] Abbas AM, Manohar CS. Investigations into critical earthquake load models within deterministic and probabilistic frameworks. Earthq Eng Struct Dyn 2002; 31(4): 813-832.

[15] Abbas AM, Manohar CS. Critical spatially-varying earthquake load models for extended structures. J Struct Engrg (JoSE, India) 2002; 29(1): 39-52.

[16] Abbas AM, Manohar CS. Reliability-based vector nonstationary random critical earthquake excitations for parametrically excited systems. Struct Safety 2007; 29: $32-48$.

[17] Takewaki I. A new method for nonstationary random critical excitation. Earthq Engrg Struct Dyn 2001; 30(4): 519-535.

[18] Takewaki I. Seismic critical excitation method for robust design: A review. J Struct Engrg ASCE 2002; 128(5): 665-672.

[19] Takewaki I. Critical envelope functions for non-stationary random earthquake input. Computers \& Structures 2004; 82(20-21): 1671-1683. 
[20] Takewaki I. Bound of earthquake input energy. J Struct Engrg ASCE 2004; 30(9): 1289-1297.

[21] Takewaki I. Probabilistic critical excitation method for earthquake energy input rate. $J$ Engrg Mech ASCE 2006; 132(9): 990-1000.

[22] Takewaki I. Critical Excitation Methods in Earthquake Engineering, Elsevier Science, Oxford.

[23] Sarkar A, Manohar CS. Critical cross power spectral density functions and the highest response of multi-supported structures subjected to multi-component earthquake excitations. Earthq Eng Struct Dyn 1996; 25: 303-315.

[24] Sarkar A, Manohar CS. Critical seismic vector random excitations for multiply supported structures. J Sound and Vibration 1998; 212(3): 525-546.

[25] Fujita K, Yoshitomi S, Tsuji M, Takewaki I. Critical cross-correlation function of horizontal and vertical ground motions for uplift of rigid block. Eng Struct 2008; 30(5): 1199-1213. 
Table 1 Structural member properties

\begin{tabular}{|c|c|c|}
\hline & column & beam \\
\hline Cross-section (mm) & $\square-1500 \times 1500 \times 50$ & $\mathrm{H}-1200 \times 600 \times 40 \times 32$ \\
\hline Cross-sectional area $\left(\mathrm{mm}^{2}\right)$ & $2.90 \times 10^{5}$ & $8.57 \times 10^{4}$ \\
\hline Second moment of area $\left(\mathrm{mm}^{4}\right)$ & $1.02 \times 10^{11}$ & $1.99 \times 10^{10}$ \\
\hline Mass per unit length $(\mathrm{kg} / \mathrm{m})$ & 2273 & 673 \\
\hline
\end{tabular}

Table 2 Geometrical and structural parameters

\begin{tabular}{|c|c|}
\hline Span length $(\mathrm{m})$ & $L_{2}=15.0$ \\
\hline horizontal stiffness $k_{1}(\mathrm{~N} / \mathrm{mm})$ & $7.62 \times 10^{8}$ \\
\hline horizontal stiffness $k_{2}(\mathrm{~N} / \mathrm{mm})$ & $7.62 \times 10^{8}$ \\
\hline mass $m_{1}(\mathrm{~kg})$ & $3.87 \times 10^{6}$ \\
\hline mass $m_{2}(\mathrm{~kg})$ & $3.87 \times 10^{6}$ \\
\hline horizontal natural period $T_{1}(\mathrm{~s})$ & 0.448 \\
\hline horizontal natural period $T_{2}(\mathrm{~s})$ & 0.448 \\
\hline
\end{tabular}




\section{Captions of Figures and Tables}

Table 1 Structural member properties

Table 2 Geometrical and structural parameters

Fig.1 Comparison of extended P-W model with P-W model

Fig.2 Coherence function of 2DGM with various auto PSD ratios with respect to various incident angles in the Penzien-Watabe model

Fig.3 One-story one-span plane frame consisting of beam of wide-flange cross-section and column of square-tube cross-section

Fig.4 Schematic illustration of the present critical excitation problem

Fig.5 Schematic diagram of the proposed procedure (order interchange of double maximization procedure including sub-problem optimization)

Fig.6 Relationship between numerical analysis of Section 5.1 and 5.2

Fig.7 Envelope function of horizontal ground motion

Fig.8 Three combinations of the auto PSD functions of 2DGM along the building structural axes with the constraint of sum of the auto PSD functions

Fig.9 Comparison of the response to the critically correlated 2DGM of various auto PSD function ratios with the responses to other inputs

Fig.10 Increase ratio of critical response from SRSS response

Fig.11 Co-spectrum and quad-spectrum for $\gamma=1$ and $L_{1}=25 \mathrm{~m}$

Fig.12 Root-mean-square extreme-fiber stress of column with respect to the angle between the two sets of axes to the critically correlated 2DGM and to the uncorrelated 2DGM (various auto PSD function ratios along the principal axes of ground motions)

Fig.13 One sample set of Monte Carlo simulation of the 2DGM; (a) critically correlated, (b) perfectly correlated

Fig.14 Comparison of the column-end extreme-fiber stress to the critically correlated 2DGM with that to the perfectly correlated ones

Fig.15 (a) Acceleration records of El Centro NS and EW during Imperial Valley 1940,

(b) Cross PSD function (co-spectrum and quad-spectrum), (c) Coherence function

Fig.16 (a) Acceleration records of SCT1 EW and NS during Mexico Michoacan 1985,

(b) Cross PSD function (co-spectrum and quad-spectrum), (c) Coherence function

Fig.17 (a) Critical-type ground motion in recent earthquake near nuclear reactor facilities,

(b) Corresponding theoretical one predicted before its occurrence 


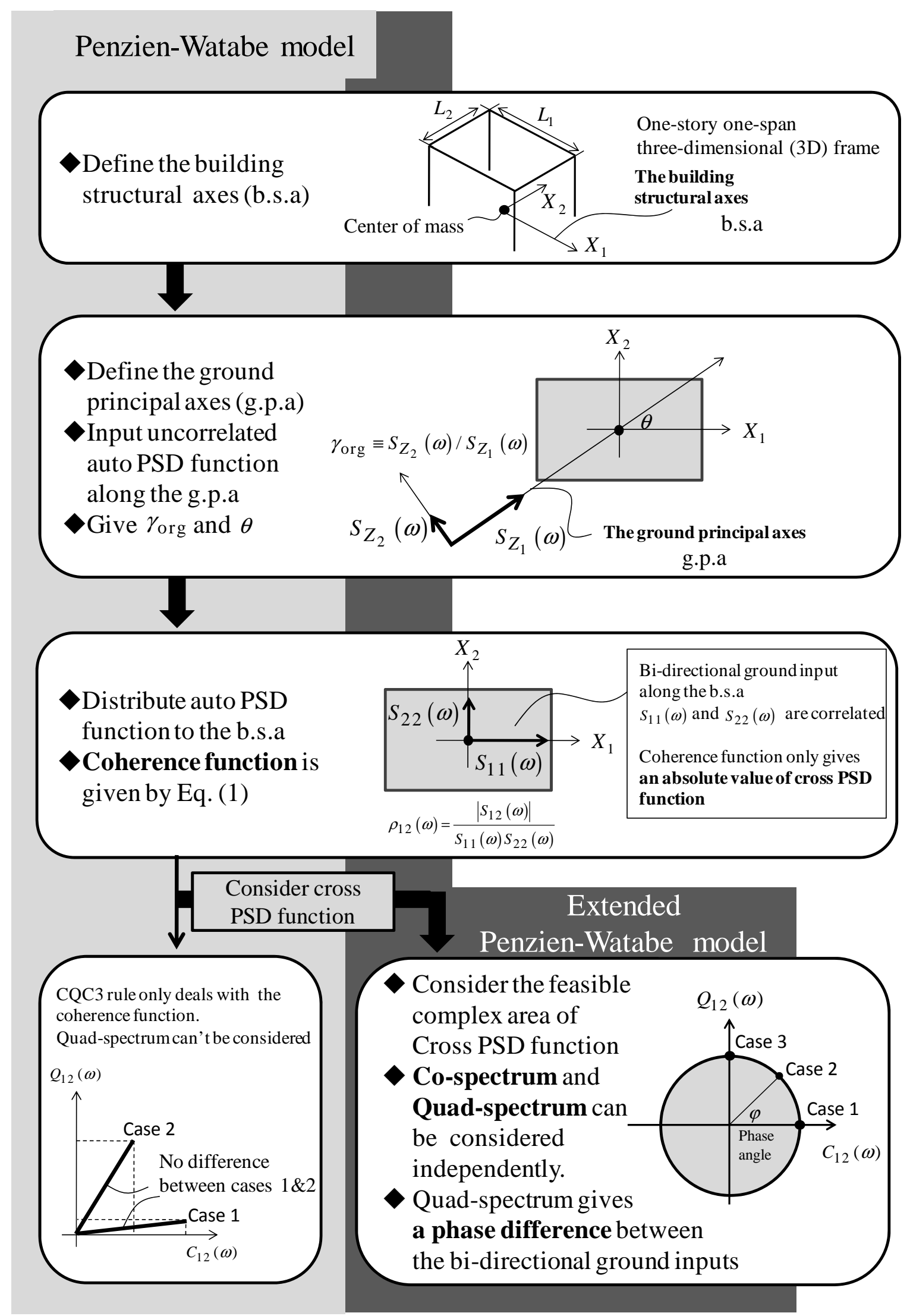

Fig.1 Comparison of extended P-W model with P-W model 


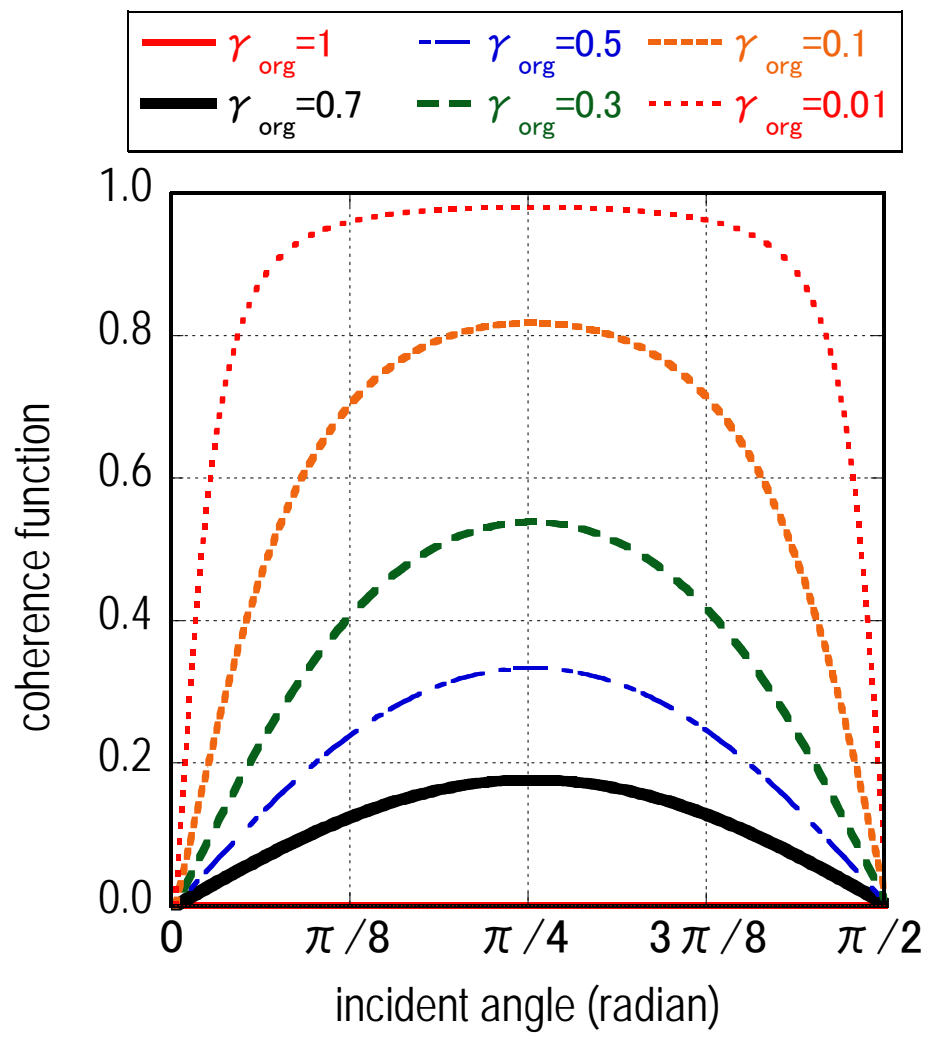

Fig.2 Coherence function of 2DGM with various auto PSD ratios with respect to various incident angles in the Penzien-Watabe model

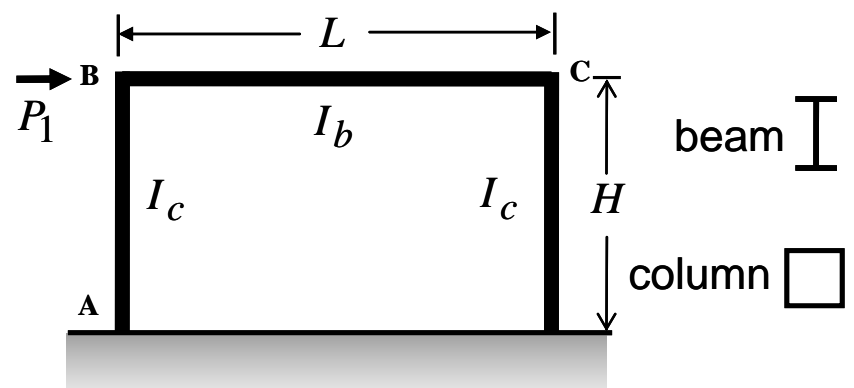

Fig.3 One-story one-span plane frame consisting of beam of wide-flange cross-section and column of square-tube cross-section 


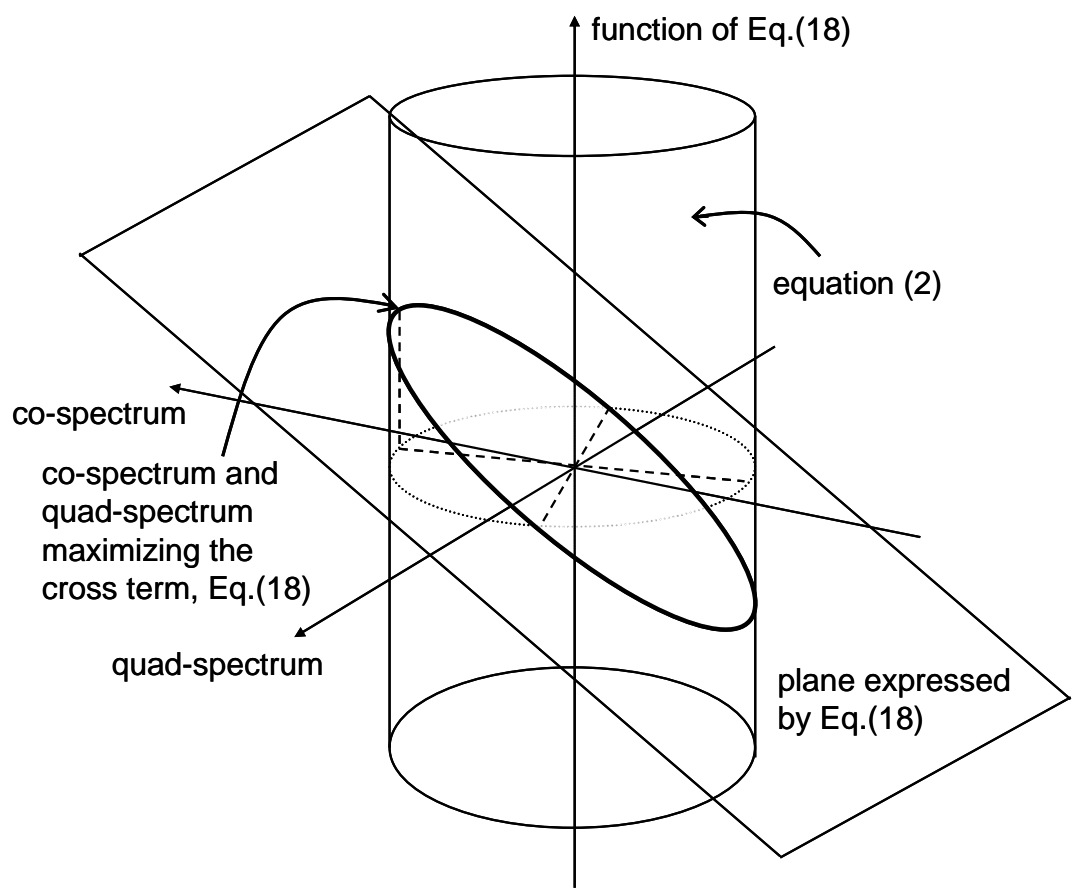

Fig.4 Schematic illustration of the present critical excitation problem
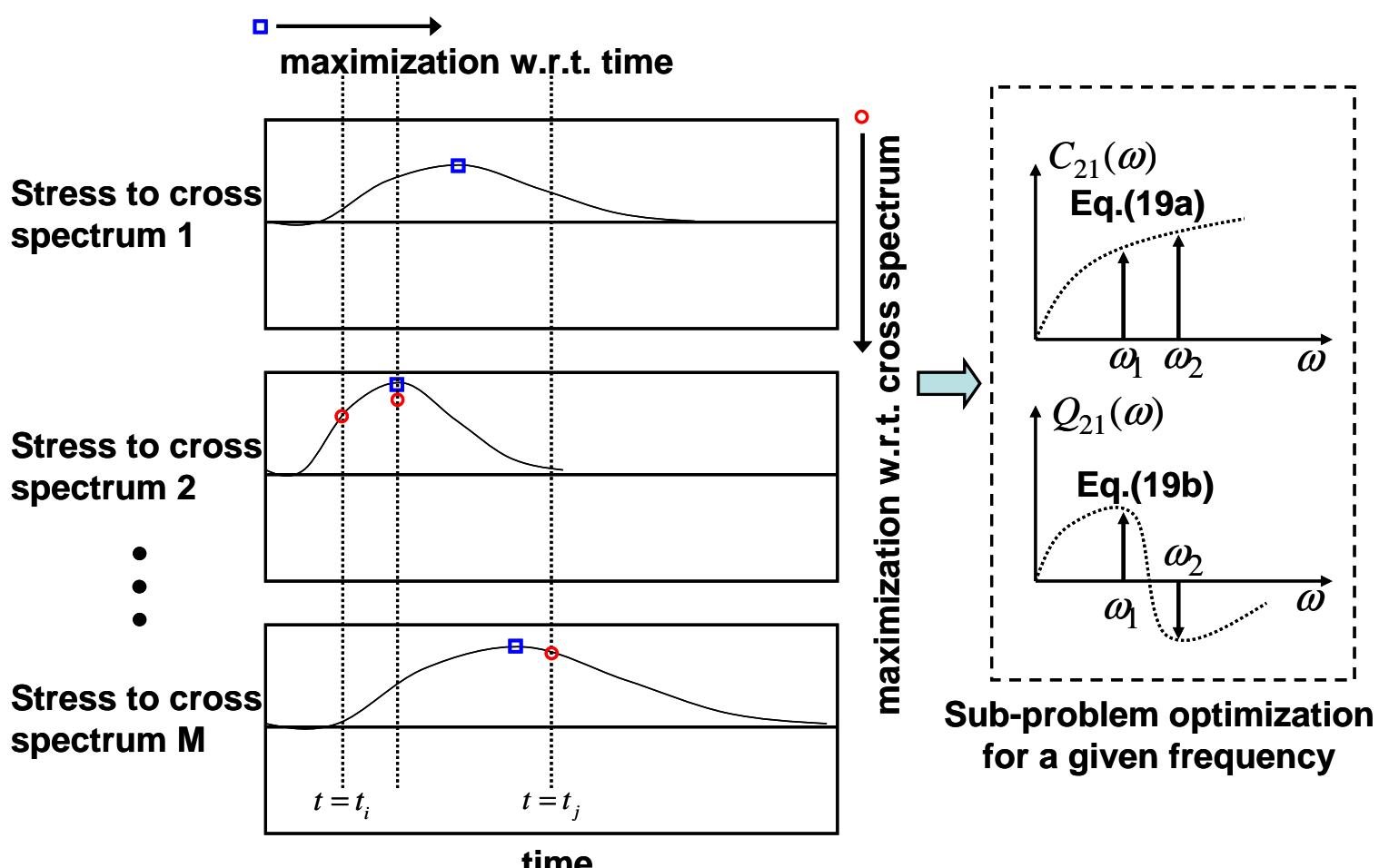

Fig.5 Schematic diagram of the proposed procedure (order interchange of double maximization procedure including sub-problem optimization) 


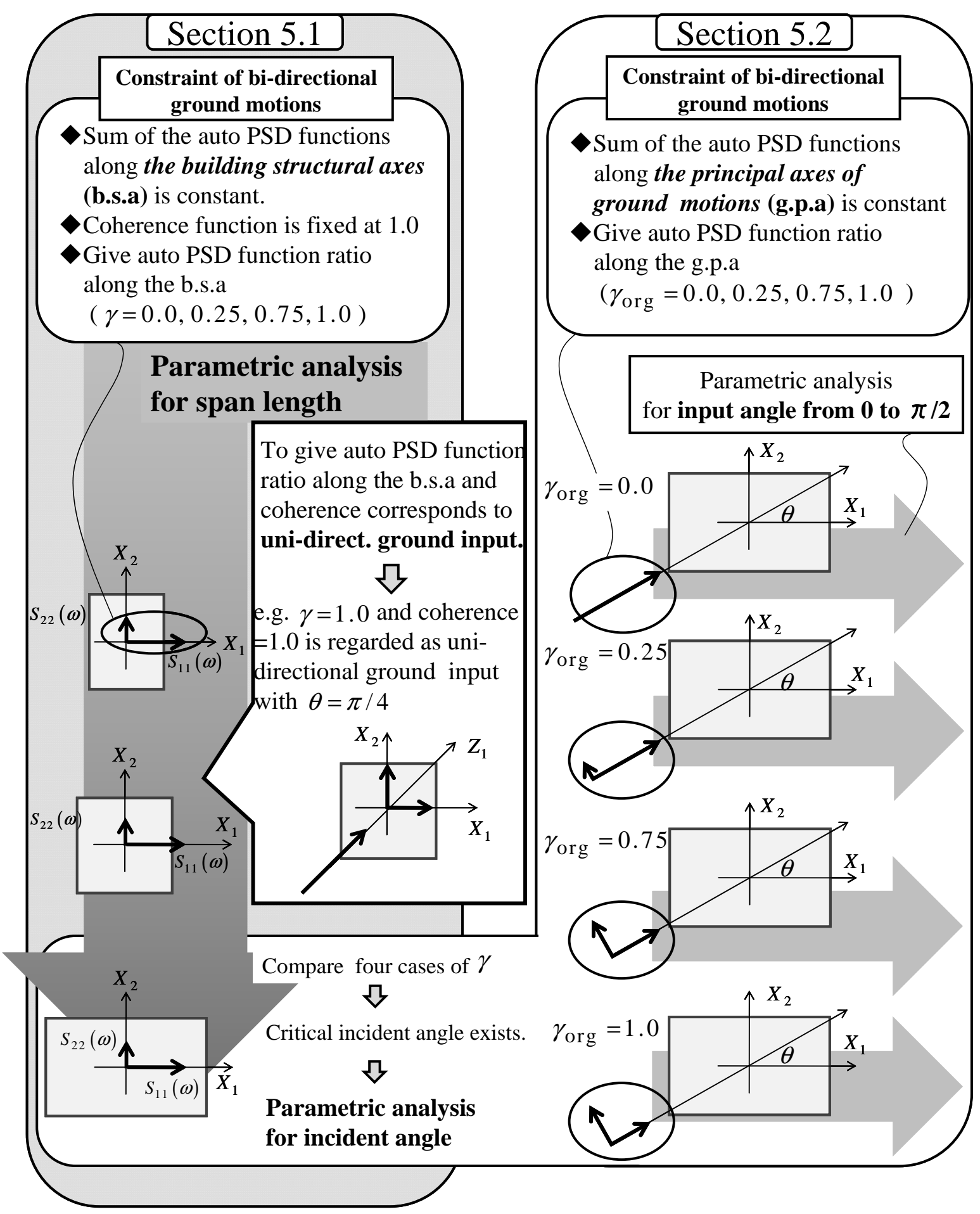

Fig.6 Relationship between numerical analysis of Section 5.1 and 5.2 


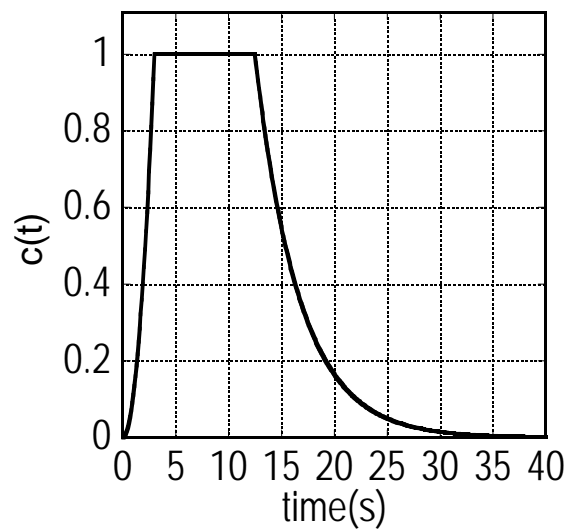

Fig.7 Envelope function of horizontal ground motion

(a)

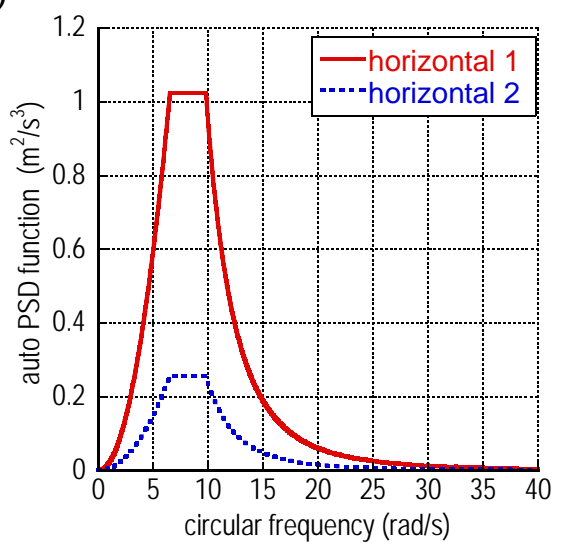

Auto PSD function ratio $\gamma=0.25$ (b)

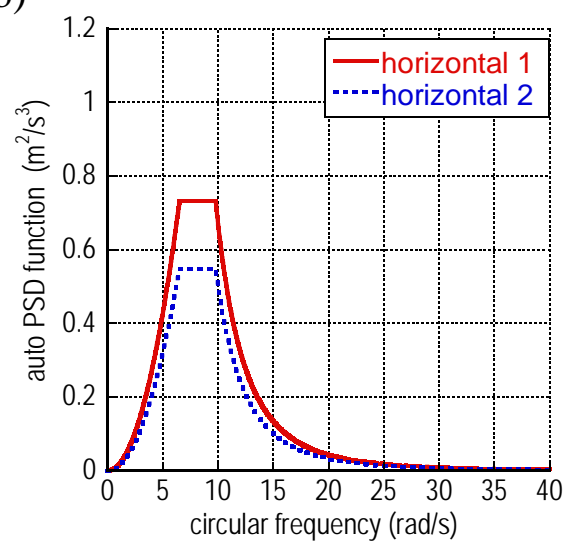

Auto PSD function ratio $\gamma=0.75$

(c)

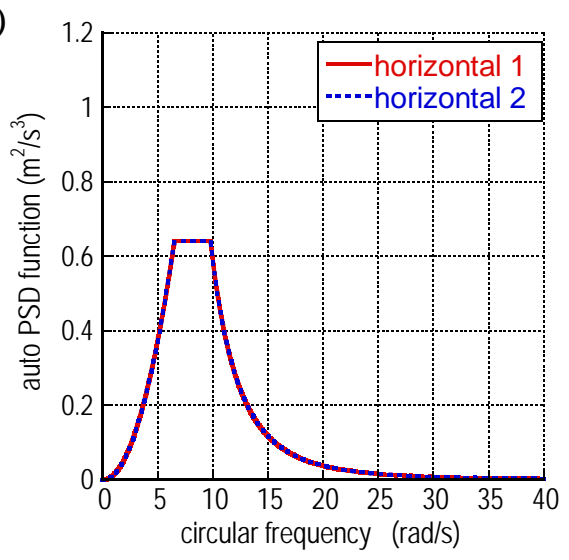

Auto PSD function ratio $\gamma=1.0$

Fig.8 Three combinations of the auto PSD functions of 2DGM along the building structural axes with the constraint of sum of the auto PSD functions 


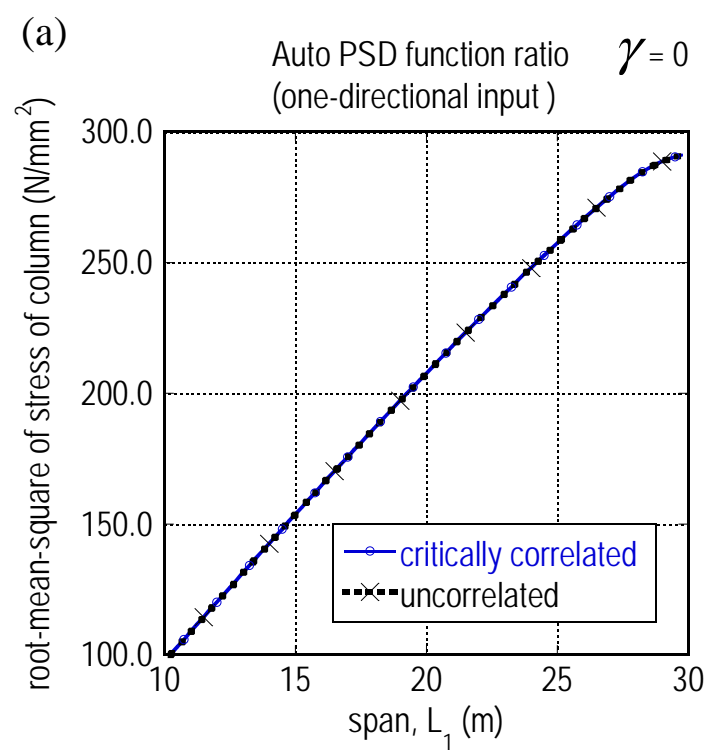

(b)

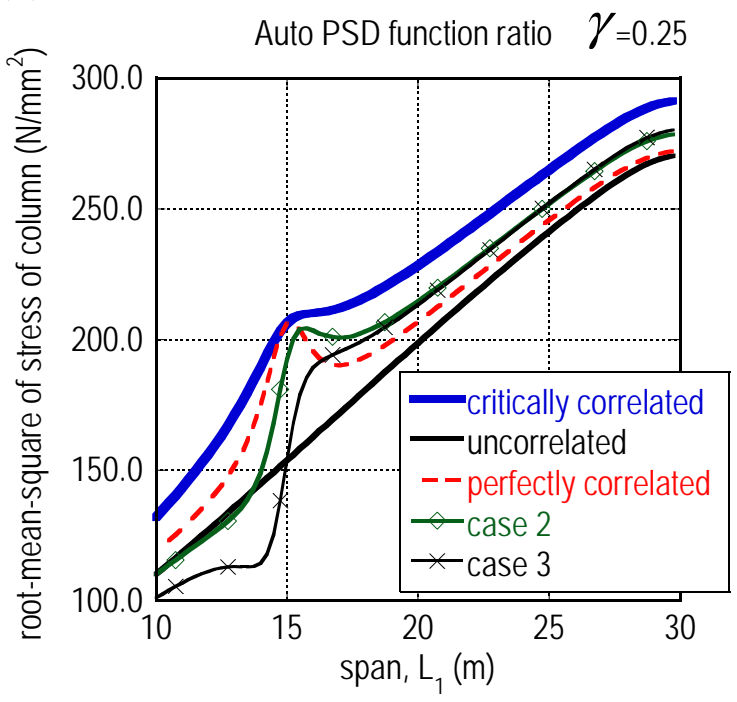

(c)

(d)
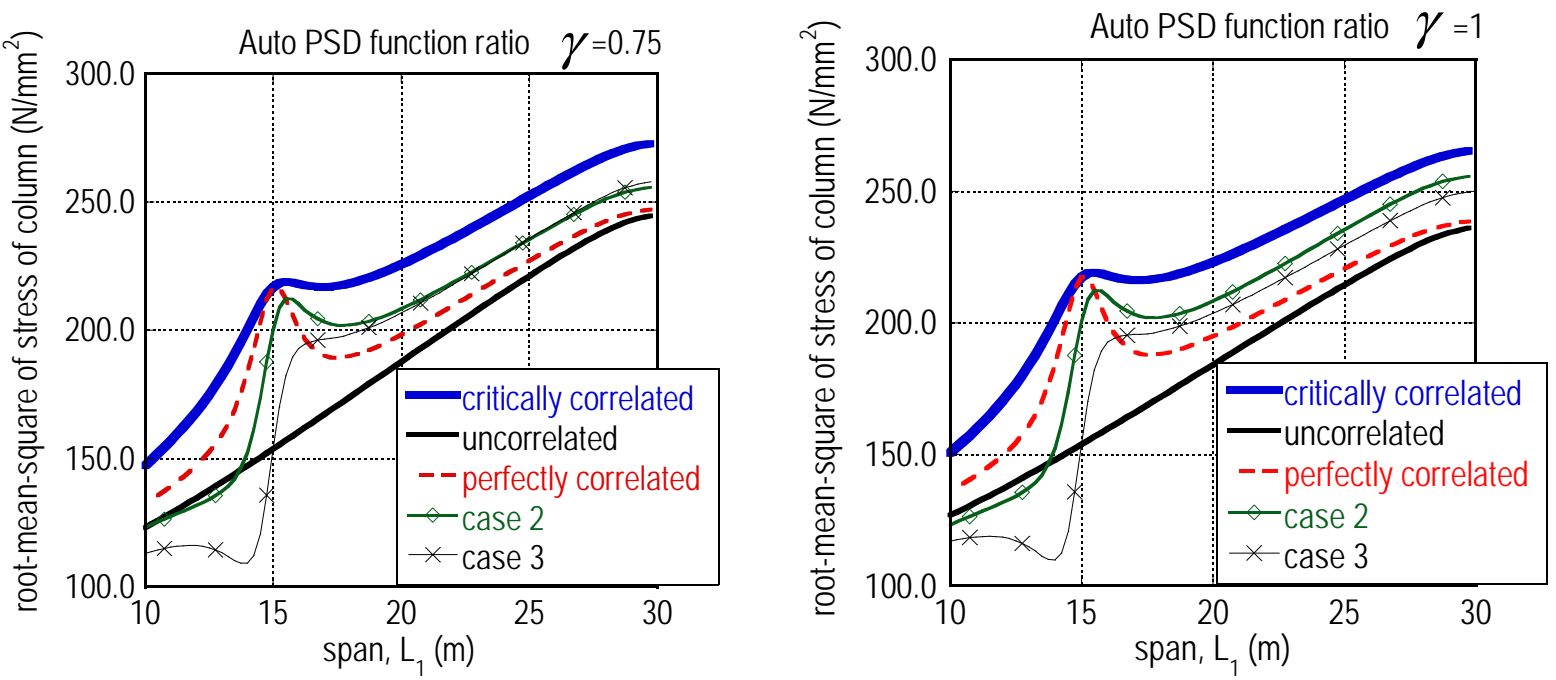

Fig.9 Comparison of the response to the critically correlated 2DGM of various auto PSD function ratios with the responses to other inputs 


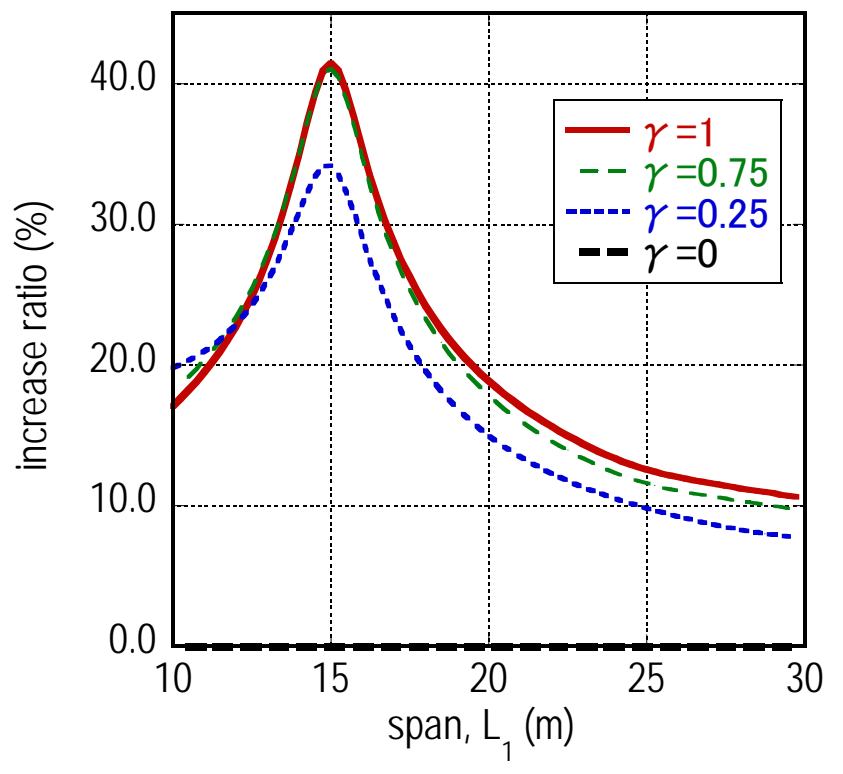

Fig.10 Increase ratio of critical response from SRSS response 


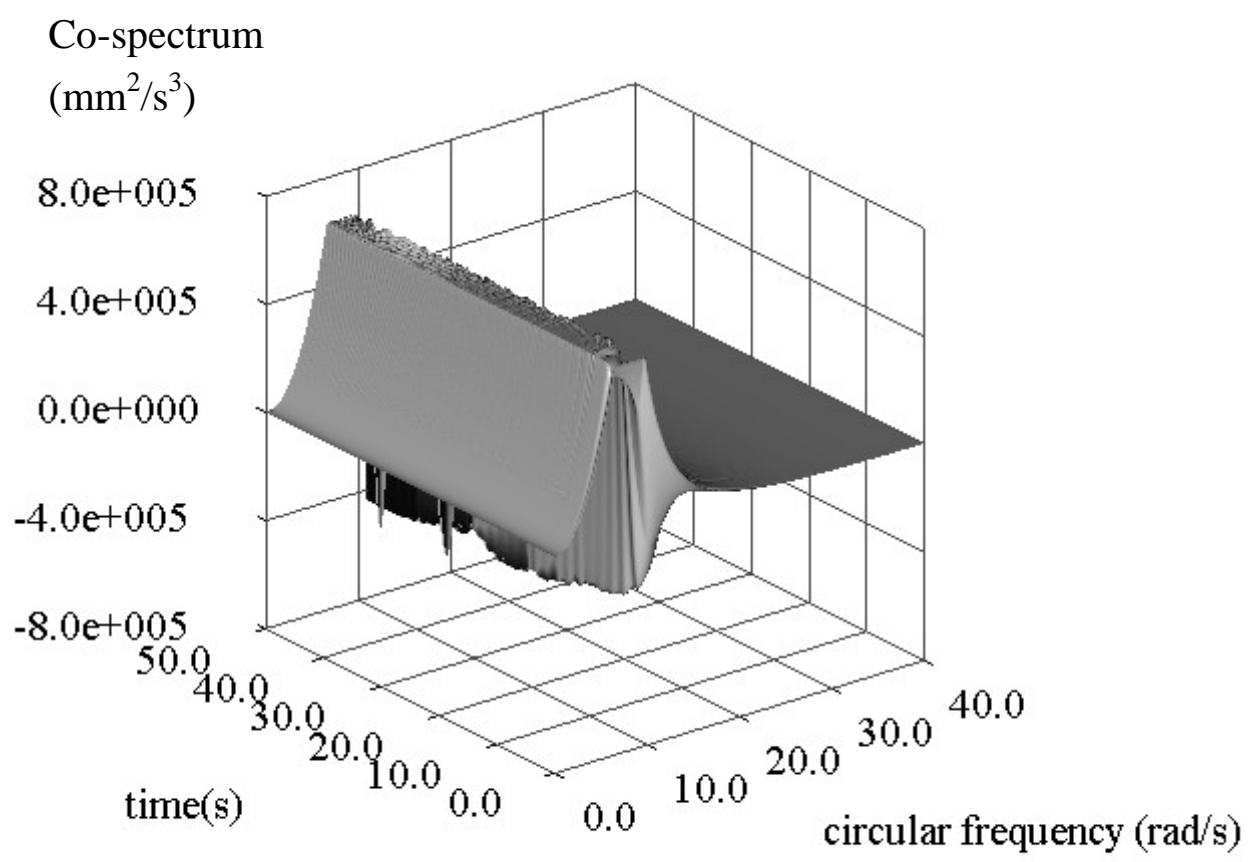

co-spectrum

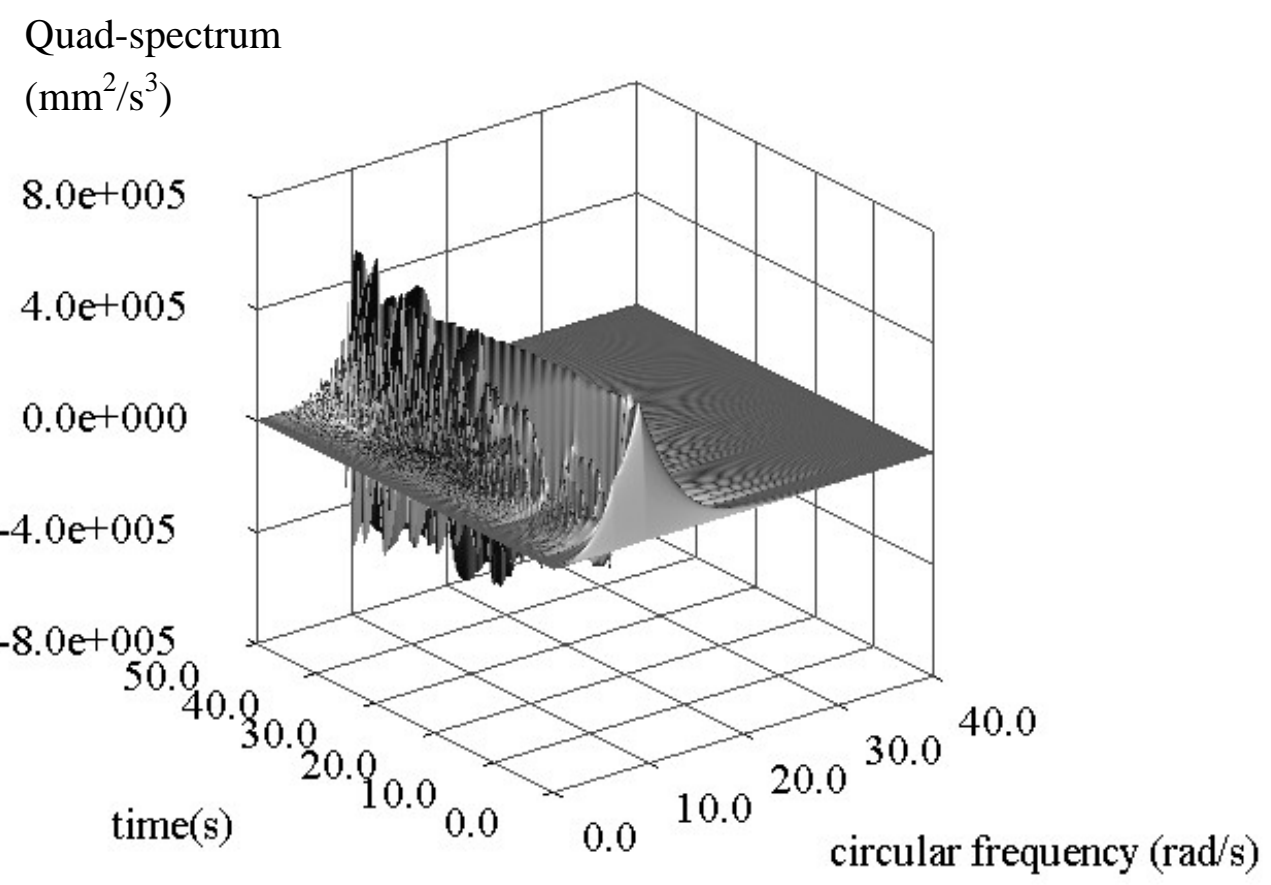

quad-spectrum

Fig.11 Co-spectrum and quad-spectrum for $\gamma=1$ and $L_{1}=25 \mathrm{~m}$ 
(a)

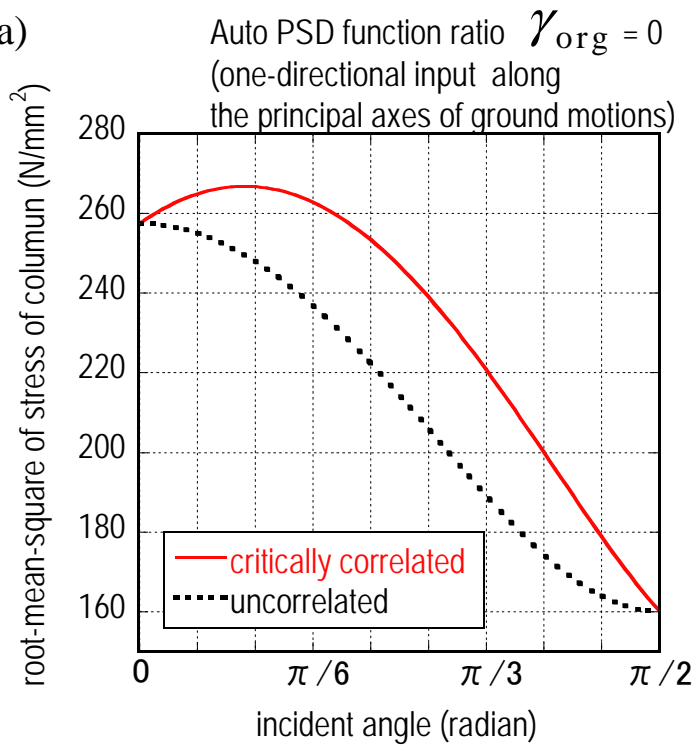

(c)

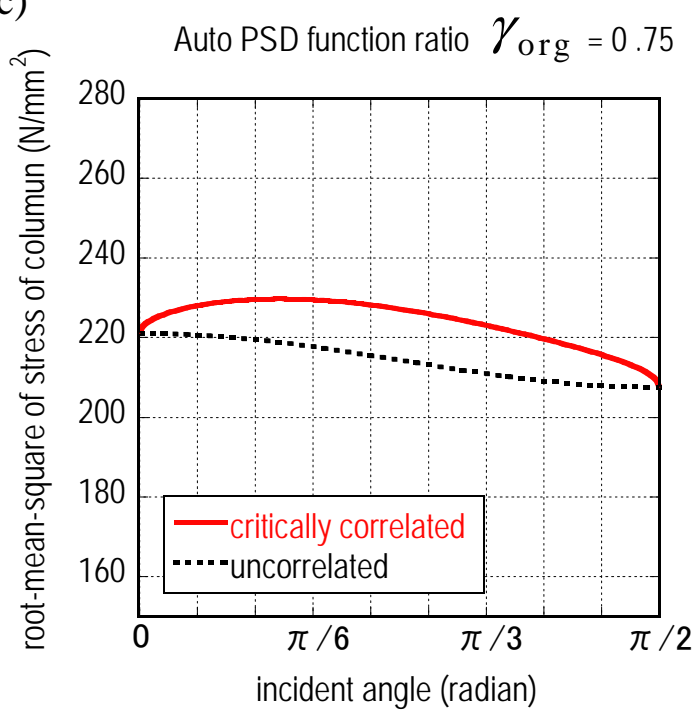

(b)

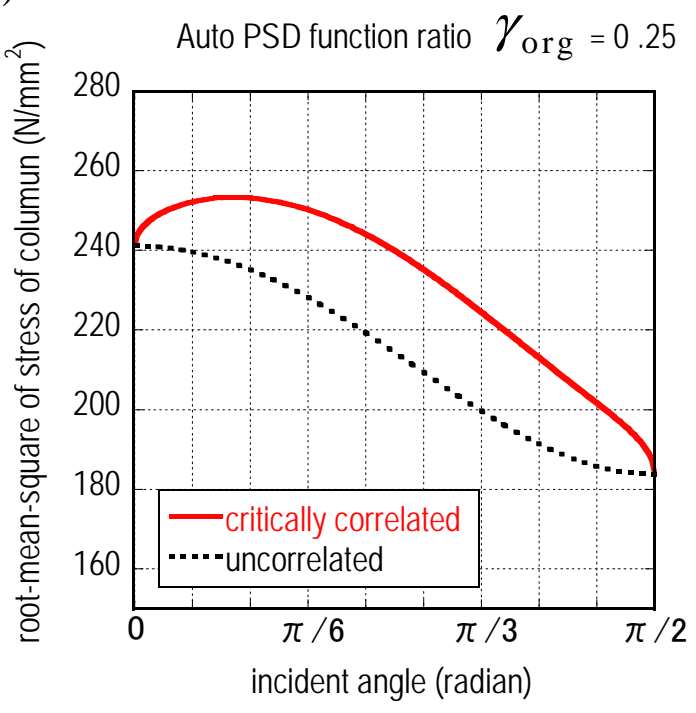

(d)

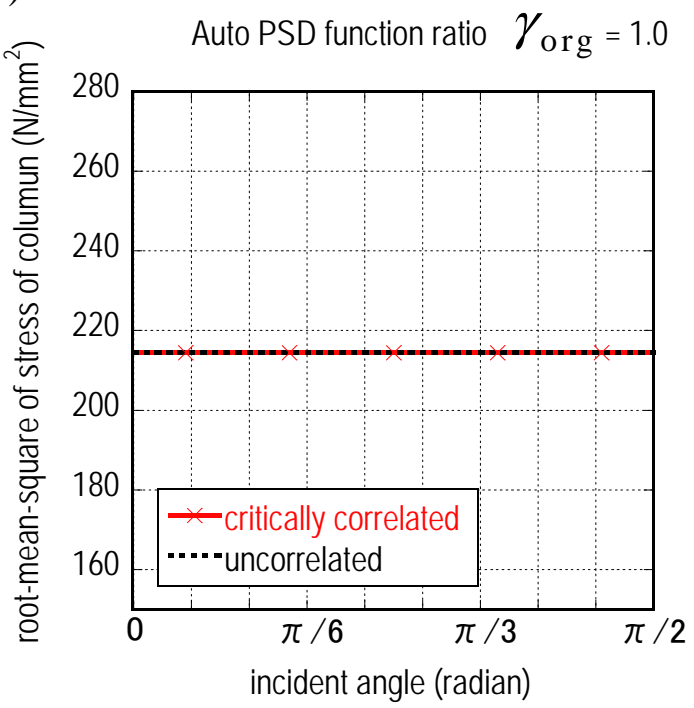

Fig.12 Root-mean-square extreme-fiber stress of column with respect to the angle between the two sets of axes to the critically correlated 2DGM and to the uncorrelated 2DGM (various auto PSD function ratios along the principal axes of ground motions) 
(a)

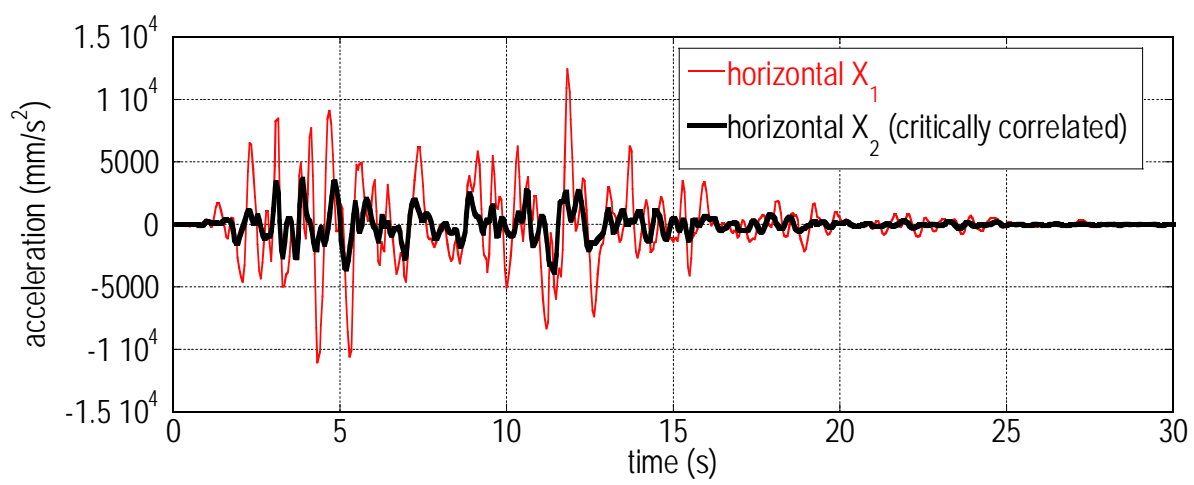

(b)

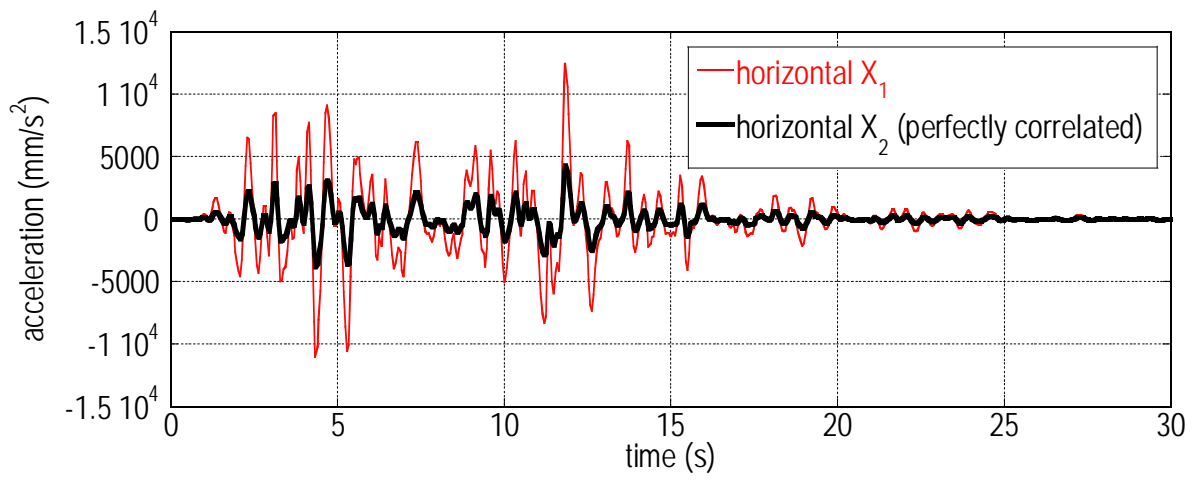

Fig.13 One sample set of Monte Carlo simulation of the 2DGM; (a) critically correlated, (b) perfectly correlated

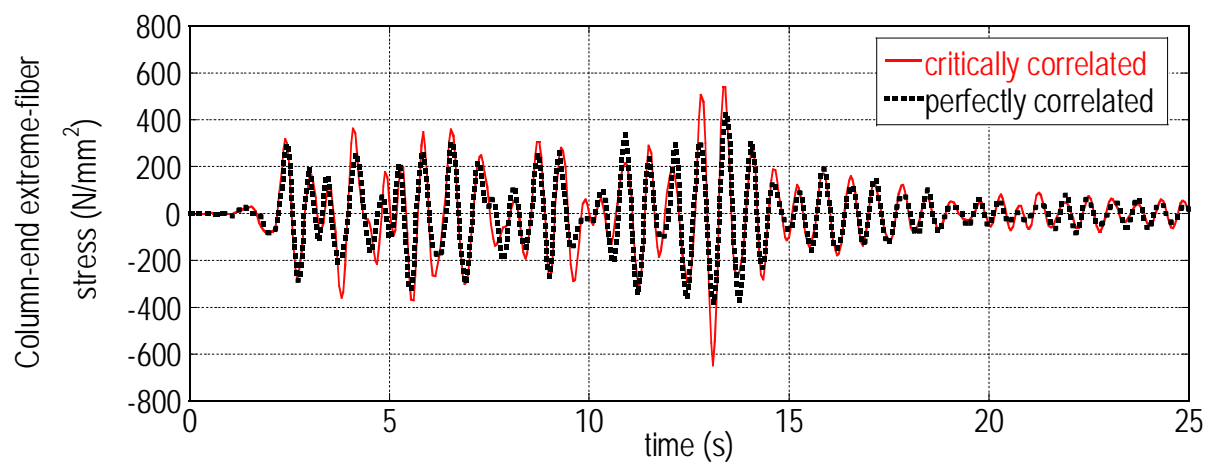

Fig.14 Comparison of the column-end extreme-fiber stress to the critically correlated 2DGM with that to the perfectly correlated ones 
(a)

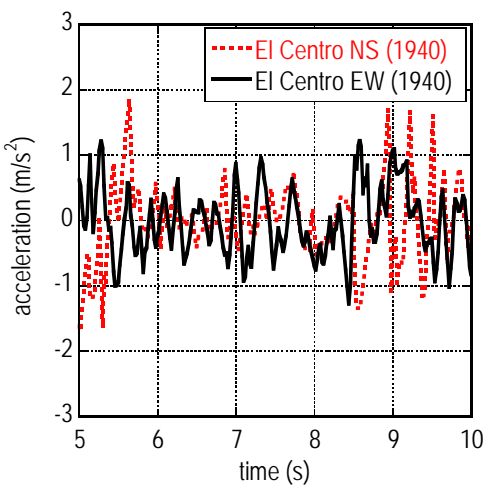

(b)

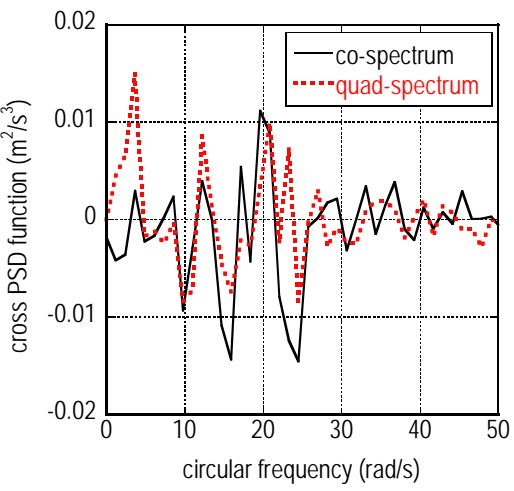

(c)

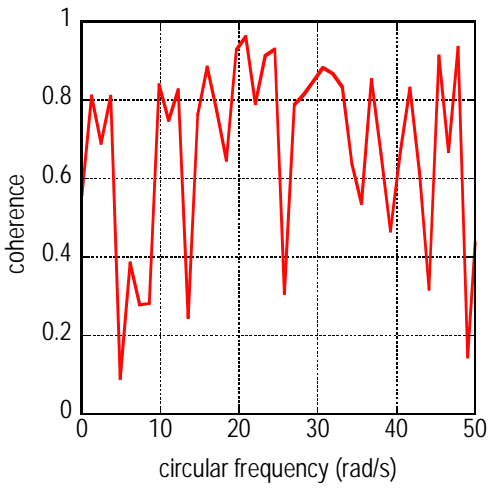

Fig.15 (a) Acceleration records of El Centro NS and EW during Imperial Valley 1940, (b) Cross PSD function (co-spectrum and quad-spectrum), (c) Coherence function

(a)

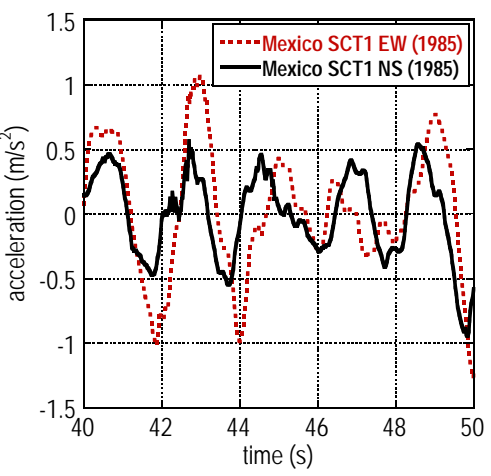

(b)

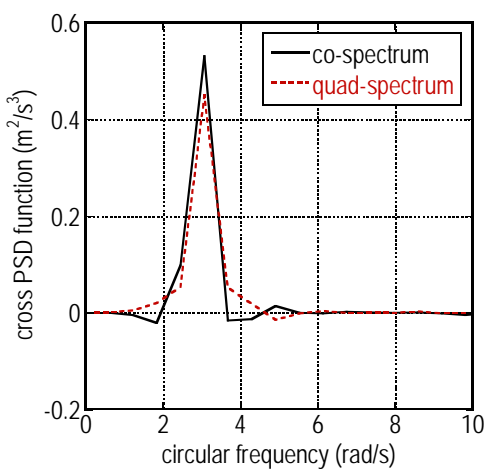

(c)

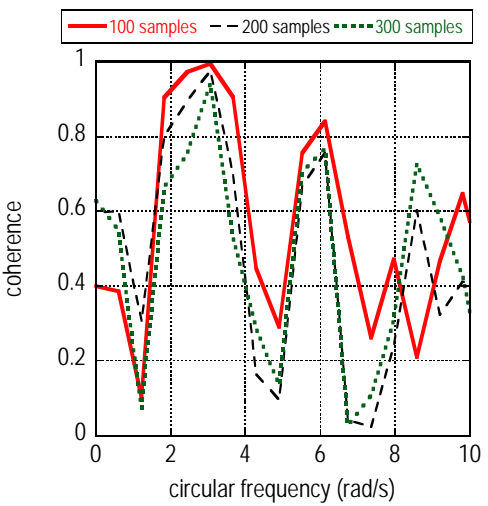

Fig.16 (a) Acceleration records of SCT1 EW and NS during Mexico Michoacan 1985, (b) Cross PSD function (co-spectrum and quad-spectrum), (c) Coherence function

(a)

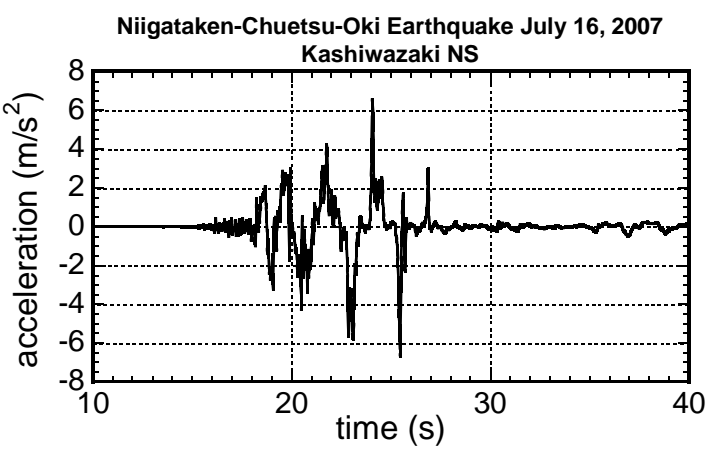

(b)

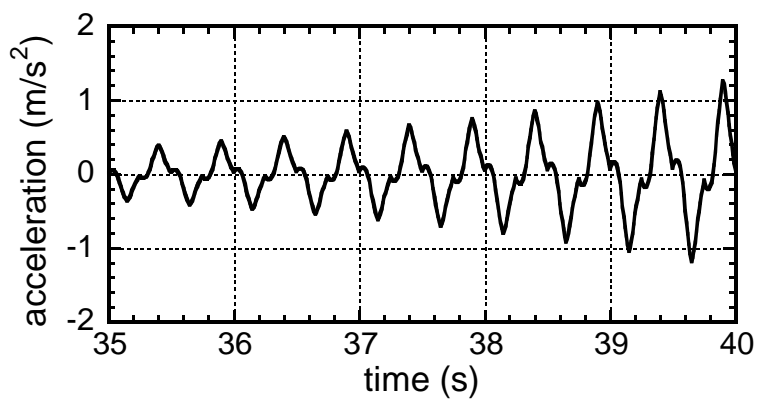

Fig.17 (a) Critical-type ground motion in recent earthquake near nuclear reactor facilities, (b) Corresponding theoretical one predicted before its occurrence 